\title{
Schwarzschild-Tangherlini metric from scattering amplitudes in various dimensions
}

\author{
Stavros Mougiakakos $\oplus^{*}$ \\ Institut de Physique Théorique, Université Paris Saclay, CNRS, F-91191 Gif-sur-Yvette, France \\ Pierre Vanhove ${ }^{\dagger}$ \\ Institut de Physique Théorique, Université Paris Saclay, CNRS, F-91191 Gif-sur-Yvette, France \\ and National Research University Higher School of Economics, 20 Myasnitskaya ulitsa, Moscow 101000, Russia, \\ and Theoretical Physics Department, CERN, 1211 Geneva 23, Switzerland
}

(Received 1 November 2020; accepted 7 December 2020; published 4 January 2021)

\begin{abstract}
We derive the static Schwarzschild-Tangherlini metric by extracting the classical contributions from the multiloop vertex functions of a graviton emitted from a massive scalar field. At each loop order the classical contribution is proportional to a unique master integral given by the massless sunset integral. By computing the scattering amplitudes up to three-loop order in general dimension, we explicitly derive the expansion of the metric up to the fourth post-Minkowskian order $O\left(G_{N}^{4}\right)$ in four, five and six dimensions. There are ultraviolet divergences that are cancelled with the introduction of higher-derivative nonminimal couplings. The standard Schwarzschild-Tangherlini is recovered by absorbing their effects by an appropriate coordinate transformation induced from the de Donder gauge condition.
\end{abstract}

DOI: 10.1103/PhysRevD.103.026001

\section{INTRODUCTION}

General relativity is a theory for the action of gravity in space and time. The dynamics of the gravitational field is constrained by the Einstein's classical field equations. They are tensorial nonlinear equations, because of the self-interaction of the gravitational field, notoriously difficult to solve. It is therefore important to develop efficient methods for studying gravity in various regimes.

General relativity can be embedded in quantum theory where the gravitational force results from the exchange of a quantized massless spin-2 graviton field [1-5]. One can then consider the Einstein-Hilbert term as the first term of a low-energy effective action containing an infinite number of higher derivative operators [6].

The classical limit $\hbar \rightarrow 0$ has been studied by Duff in [7] where he showed how to reproduce the classical Schwarzschild metric in four dimensions from quantum tree graphs up to the second order $O\left(G_{N}^{2}\right)$ in Newton's constant.

\footnotetext{
*stavros.mougiakakos@ipht.fr

pierre.vanhove@ipht.fr
}

Published by the American Physical Society under the terms of the Creative Commons Attribution 4.0 International license. Further distribution of this work must maintain attribution to the author(s) and the published article's title, journal citation, and DOI. Funded by SCOAP ${ }^{3}$.
The relation between the quantum theory of gravity and the classical Einstein's theory of general relativity has received a new interpretation with the understanding [8-13] that an appropriate (and subtle) $\hbar \rightarrow 0$ limit of quantum multiloop scattering gravitational amplitudes lead to higher $G_{N}$-order classical gravity contributions. Considering the importance of such approach for the evaluation of the post-Minkowskian expansion for the gravitational two-body scattering [14-20], we use the procedure given in [12] for extracting the classical contributions from the multiloop vertex function of a graviton emission from a massive scalar field to recover the Schwarzschild-Tangherlini metric in various dimensions. The scattering amplitude approach works in general dimensions [21-24] and gives the opportunity to explore general relativity in higher-dimensions $[25,26]$. At tree-level and one-loop our results agree with the general dimension results in $[21,24]$. We show how to reconstruct the metric up to the fourth order $O\left(G_{N}^{4}\right)$ in Newton's constant by evaluating the scattering amplitudes up to three-loop orders.

Using the procedure designed in [12] we argue, in Sec. II A, that the classical contribution at $l$-loop order is given by the two-point $l$-loop massless sunset graphs. We verify this explicitly evaluating the classical limit of the quantum scattering amplitudes up to three-loop order.

The scattering amplitudes develop ultraviolet divergences. In Sec. IV, we show how to recover the finite static Schwarzschild-Tangherlini metric by the addition of 
nonminimal couplings given schematically by (see (88) for a precise expression)

$$
\delta^{(n)} S^{\mathrm{ct}} \sim\left(G_{N} m\right)^{\frac{2 n}{d-2}} \int d^{d+1} x \sqrt{-g} \nabla^{2(n-1)} \mathcal{R}_{\mu \nu} \partial^{\mu} \phi \partial^{\nu} \phi .
$$

In four dimensions the nonminimal couplings $\delta^{(1)} S^{\text {ct }}$ have been introduced in [27] for the analysis up to the third postMinkowskian order in the context of the world-line formalism. The relation between the world-line formalism and the amplitude approach is detailed in [20]. Higherderivative couplings with $n \geq 2$ would be needed in four dimensions from the fifth post-Minkowskian order, but they appear at lowest order in higher dimensions. Indeed, we show that in five dimensions one needs to consider higher dimensional of nonminimal couplings $\delta^{(2)} S^{\text {ct }}$ at the third post-Minkowskian order and $\delta^{(3)} S^{\text {ct }}$ at the fourth postMinkowskian. Interestingly, the metric components are finite in space-time dimensions greater or equal to six, although the stress-tensor develops ultraviolet divergences from one-loop order in odd dimensions and from two-loop order in even dimensions. These divergences are cancelled by the nonminimal couplings $\delta^{(n)} S^{\text {ct }}$. Actually, we expect that an all order computation in perturbation will require an infinite set of such nonminimal couplings.

We show that the effects of the nonminimal couplings can be reabsorbed by a coordinate transformation, and they do not affect the Schwarzschild-Tangherlini space-time geometry. Since we work in the fixed gauge de Donder gauge, we give the coordinate transformation for extracting the classical space-time metric from the scattering amplitudes in that gauge. Although general relativity is coordinate system invariant, our analysis shows that there is a preferred coordinate system when extracting the classical geometry from scattering amplitudes in the de Donder gauge. The lowest-order $n=1$ nonminimal couplings have been shown to arise from the gauge fixing in $[20,24,28]$. We will not address the question of the gauge dependence, but we remark that the choice of coordinate system (or gauge) can be critical for finding solution to Einstein's equations [29].

Since "black hole formation is a robust prediction of the general theory of relativity" [30], it is satisfying to be able to embed such classical solutions in the new understanding of the relation between general relativity and the quantum theory of gravity.

The paper is organized as follows. In Sec. II we setup the connection between the perturbation expansion vertex function for the emission a graviton from a massive scalar field and the post-Minkowskian expansion of the static metric in $d+1$ dimensions. In Sec. II A we show that the classical contribution from the multiloop amplitudes is given by the massless sunset multiloop integrals in $d$ dimensions. In Sec. II B we evaluate the master integrals. In section III we derive the metric component up to the order $O\left(G_{N}^{4}\right)$ by computing the relevant amplitudes up to three-loop order in $d+1$ dimensions. In Sec. IV we compute the nonminimal couplings required for canceling the ultraviolet divergences in the amplitude computation. In Sec. V we solve the Einstein's equations in four $(d=3)$, five $(d=4)$, and six $(d=5)$ dimensions in the de Donder gauge, and we show in Sec. VI how these results match the results derived from the amplitude computations. In Sec. VII we give an interpretation of the results in this paper. The Appendix A contains formulas for the Fourier transforms used in the text, and Appendix B the vertices for the scattering amplitude computations.

\section{THE SCHWARZSCHILD-TANGHERLINI METRIC FROM SCALAR FIELD AMPLITUDES}

The Schwarzschild metric is obtained by the gravitational scattering of a scalar field of mass $m$

$\mathcal{S}=\int d^{d+1} x \sqrt{-g}\left(\frac{R}{16 \pi G_{N}}+\frac{1}{2} g^{\mu \nu} \partial_{\mu} \phi \partial_{\nu} \phi-\frac{1}{2} m^{2} \phi^{2}\right)$.

For further reference Newton's constant has length dimensions $\left[G_{N}\right]=(\text { length })^{d-1}$, the scalar field has dimension $[\phi]=(\text { length })^{1-d}$ and the mass $[m]=(\text { length })^{-1}$. We work with the mostly negative signature $(+,-, \cdots,-)$ metric.

The graviton emission from a scalar particle of mass $p_{1}^{2}=p_{2}^{2}=m^{2}$ is given by the three-point vertex function

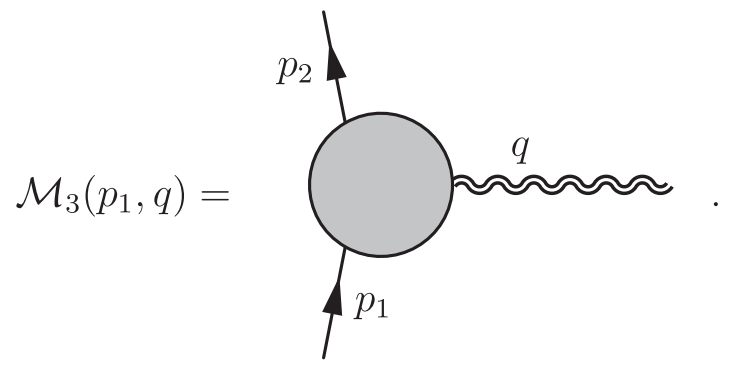

At each loop order we extract the $l$-loop contribution to the transition density of the stress-energy tensor $\left\langle T_{\mu \nu}\left(q^{2}\right)\right\rangle=\sum_{l \geq 0}\left\langle T_{\mu \nu}^{(l)}\left(q^{2}\right)\right\rangle$

$$
i \mathcal{M}_{3}^{(l)}\left(p_{1}, q\right)=-\frac{i \sqrt{32 \pi G_{N}}}{2}\left\langle T^{(l) \mu \nu}\left(q^{2}\right)\right\rangle \epsilon_{\mu \nu}
$$

where $\epsilon^{\mu \nu}$ is the polarization of the graviton with momentum $q=p_{1}-p_{2}$ is the momentum transfer.

The scattering amplitude computation is not done in the harmonic gauge coordinates $g^{\mu \nu} \Gamma_{\mu \nu}^{\lambda}(g)=0$ but in the de Donder gauge coordinate system [2,19,21,24,27]

$$
\eta^{\mu \nu} \Gamma_{\mu \nu}^{\lambda}(g)=\eta^{\mu \nu} g^{\lambda \rho}\left(\frac{\partial g_{\rho \mu}}{\partial x^{\nu}}+\frac{\partial g_{\rho \nu}}{\partial x^{\mu}}-\frac{\partial g_{\mu \nu}}{\partial x^{\rho}}\right)=0,
$$


the metric perturbations $g_{\mu \nu}=\eta_{\mu \nu}+\sum_{n \geq 1} h_{\mu \nu}^{(n)}$ satisfy $^{1}$

$$
\frac{\partial}{\partial x^{\lambda}} h_{\nu}^{\lambda(n)}-\frac{1}{2} \frac{\partial}{\partial x^{\nu}} h^{(n)}=0 .
$$

The de Donder gauge relation between the metric perturbation and the stress-energy tensor reads

$$
\begin{aligned}
h_{\mu \nu}^{(l+1)}(\vec{x})= & -16 \pi G_{N} \int \frac{d^{d} \vec{q}}{(2 \pi)^{d}} e^{i \vec{q} \cdot \vec{x}} \frac{1}{\vec{q}^{2}} \\
& \times\left(\left\langle T_{\mu \nu}^{(l)}\right\rangle^{\text {class }}\left(q^{2}\right)-\frac{1}{d-1} \eta_{\mu \nu}\left\langle T^{(l)}\right\rangle^{\text {class }}\left(q^{2}\right)\right) .
\end{aligned}
$$

In this relation enters the classical contribution at $l$ loop order $\left\langle T_{\mu \nu}^{(l)}\right\rangle^{\text {class }}\left(q^{2}\right)$ defined by the classical limit of the quantum scattering amplitude $[10,12,13]$. From now, we are dropping the super-script class and just use the notation $\left\langle T_{\mu \nu}^{(l)}\right\rangle\left(q^{2}\right)$ for the classical contribution.

\section{A. The classical contribution of the amplitude}

In this section we derive the generic form of the classical contribution of the gravity amplitudes (3) in the static limit where $q=(0, \vec{q})$ and $\vec{q}^{2} \ll m^{2}$. The classical limit is obtained by taking $\hbar \rightarrow 0$ with the momentum transfer $q / \hbar$ held fixed [13].

At the $l$-loop order we have to consider the graphs

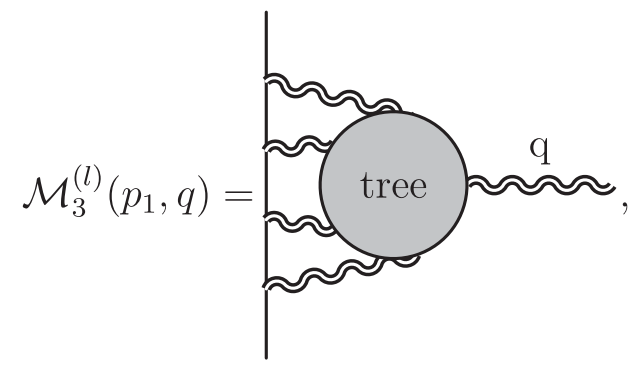

The classical contribution emerges as a particular $\hbar \rightarrow 0$ limit of the amplitude in [8,10-13]. The classical limit results in cutting the massive lines, projecting on the contribution from localized sources at different positions in space $[12,31,32]$, pictorially represented by shaded blobs

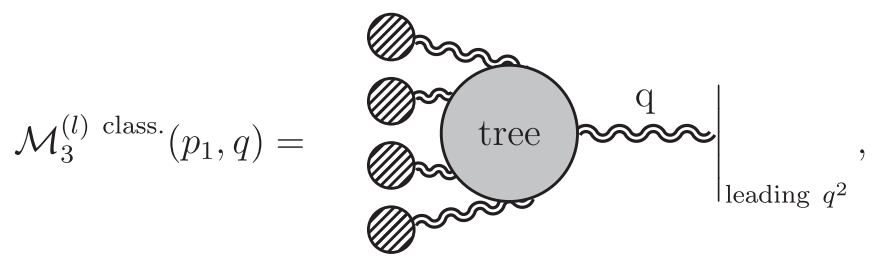

In this process one keeps only the leading $q^{2}$ contribution from the multigraviton tree-level amplitudes. The quantum tree-level graphs that were considered in [7] arise from the classical limit of the scattering amplitude up to two-loop order. In the rest of this section, we derive the generic features of the classical limit to all orders in perturbation. We then explicitly evaluate the classical limit up to three-loop order in perturbation.

The quantum amplitude in (8) is an $l+2$ gravitons amplitude with $l+1$ gravitons attached to the massive scalar line



$$
=\frac{\left(-i \sqrt{8 \pi G_{N}}\right)^{l+1} \tau_{\mu_{1} \nu_{1}}\left(p_{1}, p_{1}-\ell_{1}\right) \tau_{\mu_{2} \nu_{2}}\left(p_{1} \ell_{1}, p_{1}-\ell_{1}-\ell_{2}\right) \cdots \tau_{\mu_{l+1} \nu_{l+1}}\left(p_{1}-\ell_{1}-\cdots-\ell_{l+1}, p_{2}\right)}{\prod_{i=1}^{l}\left(\left(p_{1}-\sum_{j=1}^{i} \ell_{j}\right)^{2}-m^{2}+i \epsilon\right)},
$$

with the momentum conservation condition $\ell_{1}+\cdots+\ell_{l+1}=q=p_{1}-p_{2}$ and the vertex for emitting a graviton from a scalar field ${ }^{2}$

$$
\tau^{\mu \nu}\left(p_{1}, p_{2}\right)=p_{1}^{\mu} p_{2}^{\nu}+p_{1}^{\nu} p_{2}^{\mu}+\frac{1}{2} \eta^{\mu \nu}\left(p_{1}-p_{2}\right)^{2} .
$$

This line is attached to an $l+2$ tree-level graviton amplitude

\footnotetext{
${ }^{1}$ The harmonic gauge linearized at the first order in perturbation gives (6) with $n=1$. The higher-order expansions of the harmonic gauge differ from these conditions.

${ }^{2}$ The vertices are given in Appendix B. We have stripped of a factor $i \sqrt{8 \pi G_{N}}$ from their normalization.
} 


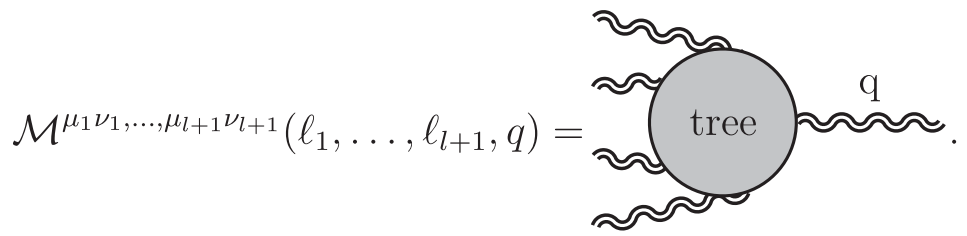

We have to sum over all the permutation of the graviton lines attached to the scalar lines. Because the gravity amplitude is invariant under the action of the permutation of the graviton lines we have

$$
\begin{aligned}
i \mathcal{M}_{3}^{(l)}\left(p_{1}, q\right)= & \frac{1}{\sqrt{4 E_{1} E_{2}}} \int \prod_{n=1}^{l} \frac{d^{d+1} \ell_{n}}{(2 \pi)^{D}}\left(\sum_{\sigma \in \mathbb{S}_{l+1}} \mathcal{L}_{\mu_{1} \nu_{1}, \ldots, \mu_{l+1} \nu_{l+1}}\left(p_{1}, p_{2}, \ell_{\sigma(1)}, \ldots, \ell_{\sigma(l+1)}\right)\right) \\
& \times \prod_{i=1}^{l+1} \frac{i \mathcal{P}^{\mu_{i} \nu_{i}, \rho_{i} \sigma_{i}}}{\ell_{i}^{2}+i \epsilon} \mathcal{M}_{\rho_{1} \sigma_{1}, \ldots, \rho_{l+1} \sigma_{l+1}}\left(\ell_{1}, \ldots, \ell_{l+1}, q\right)
\end{aligned}
$$

where $\mathfrak{S}_{l+1}$ is the group of permutation of $l+1$ elements. In the static limit the vertex (12) becomes

$$
\tau_{\mu \nu}\left(p_{1}, p_{1}-\ell\right) \simeq-2 m^{2} \delta_{\mu}^{0} \delta_{\nu}^{0}
$$

therefore the scalar line approximates to

$$
\mathcal{L}\left(p_{1}, p_{2}, \ell_{1}, \ldots, \ell_{l+1}\right) \simeq \frac{\prod_{i=1}^{l+1} i \sqrt{32 \pi G_{N}} m^{2} \delta_{\mu_{i}}^{0} \delta_{\nu_{i}}^{0}}{\prod_{i=1}^{l}\left(\left(p_{1}-\sum_{j=1}^{i} \ell_{j}\right)^{2}-m^{2}+i \epsilon\right)} .
$$

In the static limit $\left(p_{1}-L\right)^{2}-m^{2}+i \epsilon=L^{2}-2 p_{1} \cdot L+i \epsilon \simeq L_{0}^{2}-\vec{L}^{2}-2 m L_{0}+i \epsilon$. In the limit where the mass $m$ is large compared to the graviton loop momenta $|L| \ll m$ we have

$$
\begin{aligned}
L_{0}^{2}-\vec{L}^{2}-2 m L_{0}+i \epsilon & =\left(L_{0}-m-\sqrt{\vec{L}^{2}+m^{2}-i \epsilon}\right)\left(L_{0}-m+\sqrt{\vec{L}^{2}+m^{2}-i \epsilon}\right) \\
& \simeq\left(L_{0}-2 m-\frac{\vec{L}^{2}}{2 m}+i \epsilon\right)\left(L_{0}+\frac{\vec{L}^{2}}{2 m}-i \epsilon\right) \simeq-2 m\left(L_{0}-i \epsilon\right) .
\end{aligned}
$$

Therefore we have

$$
\mathcal{L}\left(p_{1}, p_{2}, \ell_{1}, \ldots, \ell_{l+1}\right) \simeq i \sqrt{32 \pi G_{N}} m^{2} \delta_{\mu_{l+1}}^{0} \delta_{\nu_{l+1}}^{0} \prod_{i=1}^{l} \frac{-i 2 \sqrt{2 \pi G_{N}} m \delta_{\mu_{i}}^{0} \delta_{\nu_{i}}^{0}}{\sum_{j=1}^{i} \ell_{j}^{0}-i \epsilon}
$$

Using momentum conservation $\ell_{1}+\cdots+\ell_{l+1}=p_{1}-p_{2}$ and that in the static limit $p_{1}^{0}-p_{2}^{0} \simeq 0$ we have

$$
\mathcal{L}\left(p_{1}, p_{2}, \ell_{1}, \ldots, \ell_{l+1}\right) \simeq 2 m i \epsilon \prod_{i=1}^{l+1} \frac{-i 2 \sqrt{2 \pi G_{N}} m \delta_{\mu_{i}}^{0} \delta_{\nu_{i}}^{0}}{\sum_{j=1}^{i} \ell_{j}^{0}-i \epsilon}
$$

Using the identity ${ }^{3}$

${ }^{3}$ This was proven in the Appendix of [33]. We give here an alternative proof using recursion. For $l=1$ we have $\Sigma(2)=\frac{1}{x_{1}\left(x_{1}+x_{2}\right)}+\frac{1}{x_{2}\left(x_{1}+x_{2}\right)}=\frac{1}{x_{1} x_{2}}$. Assuming that (22) is true at the order $l$, then at the order $l+1$ we have

$$
\Sigma(l+1)=\sum_{\sigma \in \mathbb{\Phi}_{l+1}} \prod_{i=1}^{l+1} \frac{1}{\sum_{j=1}^{i} x_{\sigma(j)}}=\frac{1}{x_{1}+\cdots+x_{l+1}} \sum_{i=1}^{l+1} \sum_{\sigma \in \mathbb{E}_{l}} \prod_{i=1}^{l} \frac{1}{\sum_{j=1}^{i} \hat{x}_{\sigma(j)}}
$$

where $\sigma(n+1)=i$ and the $\left\{\hat{x}_{1}, \ldots, \hat{x}_{l}\right\}=\left\{x_{1}, \ldots, x_{l+1}\right\} \backslash\left\{x_{i}\right\}$. By recursion hypothesis we can use the expression for $\Sigma(l)$

$$
\Sigma(l+1)=\frac{1}{x_{1}+\cdots+x_{l+1}} \sum_{i=1}^{l+1} \prod_{i=1}^{l} \frac{1}{\hat{x}_{i}}=\frac{1}{x_{1}+\cdots+x_{l+1}} \sum_{i=1}^{l+1} x_{i} \prod_{i=1}^{l+1} \frac{1}{x_{i}}=\prod_{i=1}^{l+1} \frac{1}{x_{i}} .
$$




$$
\sum_{\sigma \in \mathbb{S}_{l+1}} \prod_{i=1}^{l+1} \frac{1}{\sum_{j=1}^{i} x_{\sigma(j)}}=\prod_{i=1}^{l+1} \frac{1}{x_{i}}
$$

In the limit $\epsilon \rightarrow 0$ the expression vanishes unless some of the $\ell_{j}^{0}$ vanish at the same time. This means that one needs to pick the residues at $\ell_{j}^{0}=i \epsilon$ for $j=1, \ldots, l$ to have a nonvanishing answer. This implies that the amplitude (14) reduces to

$$
i \mathcal{M}_{3}^{(l)}\left(p_{1}, q\right) \simeq-\left.i^{l}\left(2 \sqrt{2 \pi G_{N}} m\right)^{l+1} \int \prod_{n=1}^{l} \frac{d^{d} \vec{\ell}_{n}}{(2 \pi)^{d}} \prod_{i=1}^{l+1} \frac{\mathcal{P}^{00, \rho_{i} \sigma_{i}}}{\prod_{i=1}^{l+1}\left(\ell_{i}^{2}+i \epsilon\right)} \mathcal{M}_{\rho_{1} \sigma_{1}, \ldots, \rho_{l+1} \sigma_{l+1}}\left(\ell_{1}, \ldots, \ell_{l+1}, q\right)\right|_{\ell_{i}^{0}=0}
$$

with $\ell_{1}+\cdots+\ell_{l+1}=q$. We recall that

$$
\mathcal{P}^{00, \rho \sigma}=\delta_{0}^{\rho} \delta_{0}^{\sigma}-\frac{\eta^{\rho \sigma}}{D-2} .
$$

The amplitude (23) corresponds to the graph where the scalar line has been collapsed to a point

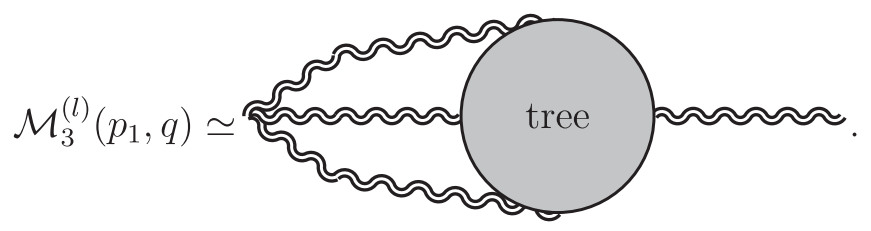

In the static with $q=(0, \vec{q}),|q| \ll m$, the $l+2$-tree level gravitons amplitude has the leading behavior

$$
\prod_{n=1}^{l+1} \mathcal{P}^{00, \rho_{i} \sigma_{i}} \mathcal{M}_{\rho_{i} \sigma_{i}, \ldots, \rho_{l+1} \sigma_{l+1}}\left(\ell_{1}, \ldots, \ell_{l+1}, q\right) \propto{\sqrt{G_{N}} l}^{l},
$$

and higher powers of $\vec{q}^{2}$ contribute to higher powers of $\hbar$ and are subleading quantum corrections (see Sec. III A for more about this).

Therefore, the classical contribution to the stress-tensor in (4) is given by ${ }^{4}$

$$
\left\langle T_{\mu \nu}^{(l)}\right\rangle=\pi^{l}\left(G_{N} m\right)^{l} m\left(c_{1}^{(l)}(d) \delta_{\mu}^{0} \delta_{\nu}^{0}+c_{2}^{(l)}(d)\left(\frac{q_{\mu} q_{\nu}}{q^{2}}-\eta_{\mu \nu}\right)\right) J_{(l)}\left(q^{2}\right)
$$

where $c_{1}^{(l)}(d)$ and $c_{2}^{(l)}(d)$ are rational functions of the dimension $d$ and $J_{(n)}\left(q^{2}\right)$ is the massless $n$-loop sunset graph

$$
J_{(n)}\left(\vec{q}^{2}\right)=q \rightarrow \begin{aligned}
& \vec{q}^{2} \\
& \prod_{i=1}^{n} \vec{l}_{i}^{2}\left(\overrightarrow{l_{1}}+\cdots+\vec{l}_{n}+\vec{q}\right)^{2}
\end{aligned} \prod_{i=1}^{n} \frac{d^{d} \vec{l}_{i}}{(2 \pi)^{d}} \text {. }
$$

\section{B. The master integrals for the classical limit}

The master integrals (28) can be evaluated straightforwardly with the parametric representation of the $n$-loop sunset in $D$ dimensions (see [36])

$$
J_{(n)}\left(\vec{q}^{2}\right)=\frac{\left(\vec{q}^{2}\right)^{\frac{n(d-2)}{2}}}{(4 \pi)^{\frac{n d}{2}}} \Gamma\left(n+1-\frac{n d}{2}\right) \int_{x_{i} \geq 0}\left(\frac{1}{x_{1}}+\cdots+\frac{1}{x_{n}}+1\right)^{\frac{(n+1)(2-d)}{2}} \prod_{i=1}^{n} \frac{d x_{i}}{x_{i}^{\frac{d}{2}}}
$$

\footnotetext{
${ }^{4}$ We have checked this explicitly to three-loop order using the Litered code $[34,35]$.
} 
since the first Symanzik polynomial is $U_{n+1}=$ $\left(\sum_{i=1}^{n+1} \frac{1}{x_{i}}\right)\left(\prod_{i=1}^{n+1} x_{i}\right)$ and the second Symanzik polynomial is $F_{n+1}=-q^{2} x_{1} \cdots x_{n+1}=\vec{q}^{2} x_{1} \cdots x_{n+1}$. Changing variables to $y_{i}=1 / x_{i}$ we have

$$
\begin{aligned}
J_{(n)}\left(\vec{q}^{2}\right)= & \frac{\left(\vec{q}^{2}\right)^{\frac{n(d-2)}{2}}}{(4 \pi)^{\frac{n d}{2}}} \Gamma\left(n+1-\frac{n d}{2}\right) \\
& \times \int_{y_{i} \geq 0}\left(y_{1}+\cdots+y_{n}+1\right)^{\frac{(n+1)(2-d)}{2}} \prod_{i=1}^{n} \frac{d y_{i}}{y_{i}^{\frac{4-d}{2}}} .
\end{aligned}
$$

Using the expression for Euler's beta-function

$$
\int_{0}^{\infty}(x+a)^{\alpha} \frac{d x}{x^{1-\beta}}=a^{\alpha+\beta} \frac{\Gamma(-\beta-\alpha) \Gamma(\beta)}{\Gamma(-\alpha)},
$$

the master integral is readily evaluated to be

$$
J_{(n)}\left(\vec{q}^{2}\right)=\frac{\left(\vec{q}^{2}\right)^{\frac{n(d-2)}{2}}}{(4 \pi)^{\frac{n d}{2}}} \frac{\Gamma\left(n+1-\frac{n d}{2}\right) \Gamma\left(\frac{d-2}{2}\right)^{n+1}}{\Gamma\left(\frac{(n+1)(d-2)}{2)}\right)} .
$$

The master integrals develop ultraviolet poles at loop orders, inducing divergences in the stress-energy tensor. We will show in Sec. IV how to renormalize these divergences with the introduction of higher-derivative couplings.

\section{THE METRIC PERTURBATION FROM GRAVITON EMISSION}

Using the relation (7) between the metric perturbation and using the expression (27) for the stress-energy tensor in $d$-dimension in the static limit we have

$$
\begin{aligned}
h_{\mu \nu}^{(l+1)}(\vec{q})= & -8\left(c_{1}^{(l)}(d)\left(2 \delta_{\mu}^{0} \delta_{\nu}^{0}-\eta_{\mu \nu}\right)\right. \\
& \left.+c_{2}^{(l)}(d)\left(2 \frac{q_{\mu} q_{\nu}}{q^{2}}+(d-2) \eta_{\mu \nu}\right)\right) \\
& \times \frac{\left(\pi G_{N} m\right)^{l+1} J_{(l)}\left(\vec{q}^{2}\right)}{\vec{q}^{2}} .
\end{aligned}
$$

The static space-time components are obtained by computing the Fourier transform in $d$ dimensions

$$
h_{\mu \nu}^{(l+1)}(\vec{x})=\int_{\mathbb{R}^{d}} h_{\mu \nu}^{(l+1)}(\vec{q}) e^{i \vec{q} \cdot \vec{x}} \frac{d^{d} \vec{q}}{(2 \pi)^{d}}
$$

Using the Fourier transformations given in Appendix A, and setting $r=|\vec{x}|$, the Fourier transform of the master integrals are given by

$$
\int_{\mathbb{R}^{d}} \frac{J_{(l)}\left(\vec{q}^{2}\right)}{\vec{q}^{2}} e^{i \vec{q} \cdot \vec{x}} \frac{d^{d} \vec{q}}{(2 \pi)^{d}}=\left(\frac{\Gamma\left(\frac{d-2}{2}\right)}{4 \pi^{\frac{d}{2}}} \frac{1}{r^{d-2}}\right)^{l+1}
$$

which is finite to all loop orders. The ultraviolet divergences in the momentum space representation in (32) has been cancelled by the Fourier transform. ${ }^{5}$

The tensorial Fourier transform

$$
\begin{aligned}
& \int_{\mathbb{R}^{d}} \frac{q_{i} q_{j}}{\vec{q}^{2}} \frac{J_{(l)}\left(\vec{q}^{2}\right)}{\vec{q}^{2}} e^{i \vec{q} \cdot \vec{x}} \frac{d^{d} \vec{q}}{(2 \pi)^{d}} \\
& =\left(\frac{\Gamma\left(\frac{d-2}{2}\right)}{4 \pi^{\frac{d}{2}}} \frac{1}{r^{d-2}}\right)^{l+1} \frac{1}{2-l(d-2)} \\
& \quad \times\left(-\delta_{i j}+(l+1)(d-2) \frac{x_{i} x_{j}}{r}\right) .
\end{aligned}
$$

diverges for $l=1$ and $d=4$ and for $l=2$ and $d=3$, and are otherwise finite.

By spherical symmetry we parameterise the metric in $d+1$ dimensions

$d s^{2}=h_{0}(r, d) d t^{2}-h_{1}(r, d) d \vec{x}^{2}-h_{2}(r, d) \frac{(\vec{x} \cdot d \vec{x})^{2}}{\vec{x}^{2}}$

so that

$$
h_{i}(\vec{x})=h_{i}^{(0)}+\sum_{l \geq 1} h_{i}^{(l)}(\vec{x})
$$

with $h_{i}^{(0)}=1,1,0$ for $i=0,1,2$, the post-Minkowskian expansion of the metric components

$$
\begin{aligned}
h_{0}^{(l+1)}(r, d)= & -\frac{16}{d-1}\left((d-2) c_{1}^{(l)}(d)+c_{2}^{(l)}(d)\right)\left(\frac{\rho(r, d)}{4}\right)^{l+1}, \\
h_{1}^{(l+1)}(r, d)= & \frac{16}{d-1}\left(c_{1}^{(l)}(d)-\left(1+\frac{d-1}{2-l(d-2)}\right) c_{2}^{(l)}(d)\right) \\
& \times\left(\frac{\rho(r, d)}{4}\right)^{l+1}, \\
h_{2}^{(l+1)}(r, d)= & 16 \frac{(d-2)(l+1)}{2-l(d-2)} c_{2}^{(l)}(d)\left(\frac{\rho(r, d)}{4}\right)^{l+1}
\end{aligned}
$$

We have introduced the radial parameter

$$
\rho(r, d)=\frac{\Gamma\left(\frac{d-2}{2}\right)}{\pi^{\frac{d-2}{2}}} \frac{G_{N} m}{r^{d-2}}
$$

which is our post-Minkowskian expansion parameter. Recall that in $d+1$ dimensions the length dimension of $\left[G_{N} m\right]=(\text { length })^{d-2}$ and $\rho(r, d)$ is dimensionless.

The metric component present poles in four dimensions $(d=3)$ from two-loop order and in five dimensions

\footnotetext{
${ }^{5}$ This fact had been noticed by L. Planté in his $\mathrm{PhD}$ thesis [31].
} 
$(d=4)$ from one-loop order. Such divergences will be removed by the contribution from the nonminimal coupling contributions in section IV.

\section{A. Tree-level amplitude}

At tree-level, the only contributing diagram is

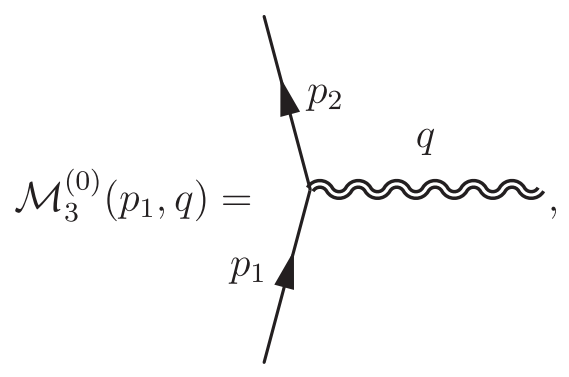

is the emission of a graviton from the scattering of two massive scalars of momenta $p_{1}$ and $p_{2}$ and $p_{1}^{2}=p_{2}^{2}=m^{2}$ with momentum transfert $q=p_{1}-p_{2}$. The scattering amplitude is given by the 2 -scalar-1-graviton vertex $\tau^{\mu \nu}\left(p_{1}, p_{2}\right)$ in $(\mathrm{B} 2)$

$$
\begin{aligned}
i \mathcal{M}_{3}^{(0)}\left(p_{1}, q\right)= & -\frac{i \sqrt{32 \pi G_{N}}}{2 \sqrt{4 E_{1} E_{2}}} \epsilon^{\mu \nu} \tau_{\mu \nu}=-\frac{i \sqrt{32 \pi G_{N}}}{2} \\
& \times \epsilon^{\mu \nu}\left(p_{1 \mu} p_{2 \nu}+p_{2 \mu} p_{1 \nu}-\eta_{\mu \nu}\left(p_{1} \cdot p_{2}-m^{2}\right)\right) .
\end{aligned}
$$

Using that $P=\left(p_{1}+p_{2}\right) / 2$ and $q=p_{1}-p_{2}$ we have that $i \mathcal{M}_{3}^{(0)}\left(p_{1}, q\right)=-\frac{i \sqrt{32 \pi G_{N}}}{2 \sqrt{4 E_{1} E_{2}}} \epsilon^{\mu \nu}\left(2 P_{\mu} P_{\nu}-\frac{1}{2}\left(q_{\mu} q_{\nu}-\eta_{\mu \nu} q^{2}\right)\right)$.

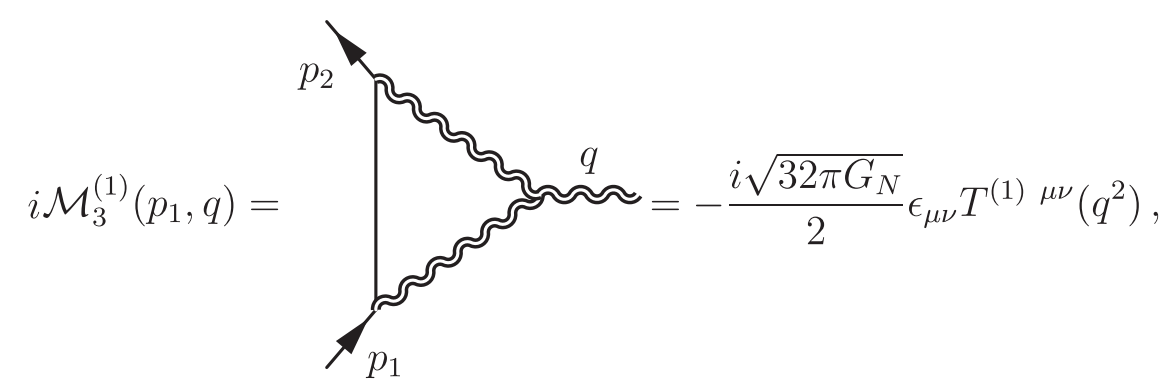

from which we extract the one-loop contribution to the stress-energy tensor in $d+1$ dimensions

$$
T^{(1) \mu \nu}\left(q^{2}\right)=\frac{i 8 \pi G_{N}}{\sqrt{4 E_{1} E_{2}}} \int \frac{d^{d+1} l}{(2 \pi)^{D}} \frac{\tau^{\sigma \rho}\left(p_{1}, l+p_{1}\right) \tau_{(3) \sigma \rho, \kappa \delta}^{\mu \nu}(l, q) \tau^{\kappa \delta}\left(p_{2}, l+p_{1}\right)}{\left(l^{2}+i \epsilon\right)\left((l+q)^{2}+i \epsilon\right)\left(\left(l+p_{1}\right)^{2}-m^{2}+i \epsilon\right)},
$$

where $\tau_{(3) \pi \rho, \sigma \tau}^{\mu \nu}\left(p_{1}, p_{2}\right)$ is the three graviton vertex and $\tau^{\mu \nu}\left(p_{1}, p_{2}\right)$ the vertex for the emission of a graviton from two scalars with momenta $p_{1}$ and $p_{2}$. We refer to appendix $\mathrm{B}$ for definitions and normalization of our vertices. 
In the static limit, $\vec{q}^{2} \ll m^{2}$, the classical contribution coming from the two scalars to one-graviton vertex is

$$
\tau_{\alpha \beta} \approx 2 m^{2} \delta_{\alpha}^{0} \delta_{\beta}^{0},
$$

using that $p_{1}^{2}=p_{2}^{2}=m^{2}$. This gives for the stress-energy tensor

$$
T^{(1) \mu \nu}\left(q^{2}\right)=i 16 \pi G_{N} m^{3} \int \frac{d^{d+1} l}{(2 \pi)^{D}} \frac{\tau_{(3) 00,00}^{\mu \nu}(l, q)}{\left(l^{2}+i \epsilon\right)\left((l+q)^{2}+i \epsilon\right)\left(\left(l+p_{1}\right)^{2}-m^{2}+i \epsilon\right)} .
$$

At this point, we want to focus on the computation of the classical contribution at the static limit. Thus, we will employ a trick, which will prove useful for higher loops. We symmetrize the diagram

$$
T^{(1) \mu \nu}\left(q^{2}\right)=i 8 \pi G_{N} m^{3} \int \frac{d^{d+1} l}{(2 \pi)^{D}} \frac{\tau_{(3) 00,00}^{\mu \nu}(l, q)}{\left(l^{2}+i \epsilon\right)\left((l+q)^{2}+i \epsilon\right)} \times\left[\frac{1}{\left(l+p_{1}\right)^{2}-m^{2}+i \epsilon}+\frac{1}{\left(l-p_{2}\right)^{2}-m^{2}+i \epsilon}\right] .
$$

In the approximation $l^{2} \ll m^{2}$ we have $\left(l+p_{i}\right)^{2}-m^{2}=l^{2}+2 l \cdot p_{1}=l^{2}+2 l_{0} E-\vec{l} \cdot \vec{q} \simeq l_{0}^{2}+2 m l_{0}$ and the amplitude reduces at leading order

$$
T^{(1) \mu \nu}\left(q^{2}\right) \simeq i 8 \pi G_{N} m^{3} \int \frac{d^{d+1} l}{(2 \pi)^{D}} \frac{\tau_{(3) 00,00}^{\mu \nu}(l, q)}{\left(l^{2}+i \epsilon\right)\left((l+q)^{2}+i \epsilon\right)}\left[\frac{1}{l_{0}^{2}+2 m l_{0}+i \epsilon}+\frac{1}{l_{0}^{2}-2 m l_{0}+i \epsilon}\right] .
$$

It is obvious that at $\mathcal{O}\left(\epsilon^{0}\right)$ order we get a zero contribution at leading order in $1 / m$, since $l_{0} \ll m$. Thus, we can compute the leading contribution of the integral over $l_{0}$ via Cauchy's theorem, by taking the residue $2 m l_{0}=i \epsilon$ and closing the contour of integration in the upper half-plane

$$
T^{(1) \mu \nu}\left(q^{2}\right)=\left.4 \pi G_{N} m^{2} \int \frac{d^{d} \vec{l}}{(2 \pi)^{d}} \frac{\tau_{(3) 00,00}^{\mu \nu}(l, q)}{\left(\vec{l}^{2}-i \epsilon\right)\left((\vec{l}+\vec{q})^{2}-i \epsilon\right)}\right|_{l_{0}=0},
$$

with

$$
\begin{aligned}
\tau_{(3) 00,00}^{\mu \nu}(l, q)= & \frac{1}{d-1}\left((d-2)\left(l^{\mu} l^{\nu}+(l+q)^{\mu}(l+q)^{\nu}+q^{\mu} q^{\nu}+\frac{3}{2} \eta^{\mu \nu} \vec{q}^{2}\right)\right. \\
& \left.-2(d-2)\left(\vec{l}_{1}^{2}+\left(\overrightarrow{l_{1}}+\vec{q}\right)^{2}\right)\left(\delta_{0}^{\mu} \delta_{0}^{\mu}-\frac{\eta^{\mu \nu}}{4}\right)-2(d-3) \vec{q}^{2} \delta_{0}^{\mu} \delta_{0}^{\mu}\right) .
\end{aligned}
$$

The component of the stress-tensor are proportional to the one-loop master integral $J_{(1)}\left(\vec{q}^{2}\right)$ as expected from the general discussion of Sec. II B

$$
\left\langle T_{\mu \nu}^{(1)}\right\rangle=\pi G_{N} m^{2}\left(c_{1}^{(1)}(d) \delta_{\mu}^{0} \delta_{\nu}^{0}+c_{2}^{(1)}(d)\left(\frac{q_{\mu} q_{\nu}}{q^{2}}-\eta_{\mu \nu}\right)\right) J_{(1)}\left(q^{2}\right)
$$

with the master integral

$$
J_{(1)}\left(q^{2}\right)=\frac{\Gamma \frac{(4-d}{2)} \Gamma\left(\frac{d-2}{2}\right)^{2}}{2^{d} \pi^{\frac{d}{2}} \Gamma(d-2)}\left(\vec{q}^{2}\right)^{\frac{d-2}{2}},
$$

and the coefficients

$$
\begin{aligned}
& c_{1}^{(1)}(d)=-\frac{2\left(4 d^{2}-15 d+10\right)}{(d-1)^{2}}, \\
& c_{2}^{(1)}(d)=-\frac{2(d-2)(3 d-2)}{(d-1)^{2}} .
\end{aligned}
$$

\footnotetext{
${ }^{6}$ One could have taken the residue at $2 m l_{0}=-i \epsilon$ and closing the contour in the lower half-plane with the same result.
} 


\section{The one-loop contribution to the metric components}

Using (39) we get for the metric components in $d+1$ dimensions

$$
\begin{aligned}
& h_{0}^{(2)}(r, d)=\frac{8(d-2)^{2}}{(d-1)^{2}} \rho(r, d)^{2}, \\
& h_{1}^{(2)}(r, d)=-\frac{4\left(2 d^{2}-9 d+14\right)}{(d-4)(d-1)^{2}} \rho(r, d)^{2}, \\
& h_{2}^{(2)}(r, d)=\frac{4(d-2)^{2}(3 d-2)}{(d-4)(d-1)^{2}} \rho(r, d)^{2},
\end{aligned}
$$

where $\rho(r, d)$ is defined in (40).
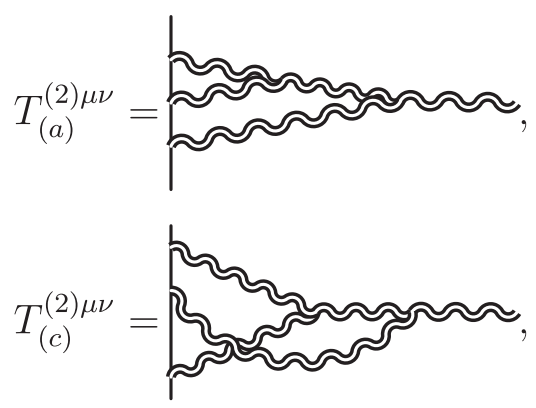

This reproduces the expression given in [21] and the expression in [[24] Eq. (22)] for $\alpha=0$.

\section{Two-loop amplitude}

The diagrams contributing to the classical corrections at third post-Minkowskian order of the metric at the two-loop graphs

$$
i \mathcal{M}_{3}^{(2)}\left(p_{1}, q\right)=-\sqrt{32 \pi G_{N}} T^{(2) \mu \nu} \epsilon_{\mu \nu}
$$

there are four contributions

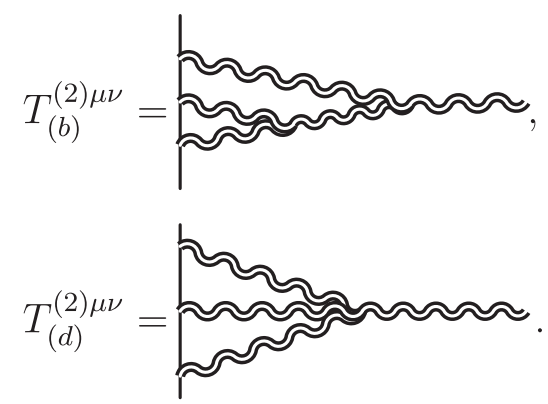

\section{The diagrams $(a),(b),(c)$}

The sum of the contributions from the diagrams $(a),(b),(c)$ after appropriate labeling of the momenta, can be expressed as

$$
\begin{aligned}
\sum_{i=a}^{c} T_{(i)}^{(2) \mu \nu}= & -\frac{16 G_{N}^{2} \pi^{2}}{m} \int \prod_{n=1}^{3} \frac{d^{d+1} l_{n}}{(2 \pi)^{2 d}} \delta\left(l_{1}+l_{2}+l_{3}+q\right) \\
& \times \frac{\tau^{\gamma \delta}\left(p_{1}, l_{1}+p_{1}\right) \tau^{\sigma \tau}\left(l_{1}+p_{1},-l_{2}+p_{1}\right) \tau^{l \theta}\left(l_{2}-p_{2},-p_{2}\right) \tau_{(3) l \theta, \sigma \tau}^{\phi \chi}\left(-l_{2}, l_{1}+q\right) \cdot \mathcal{P}_{\phi \chi} \alpha \beta \cdot \tau_{(3) \alpha \beta, \gamma \delta}^{\mu \nu}\left(l_{1}+q, q\right)}{l_{1}^{2} l_{2}^{2} l_{3}^{2}\left(l_{1}+q\right)^{2}} \\
& \times\left(\frac{1}{\left(l_{1}+p_{1}\right)^{2}-m^{2}} \frac{1}{\left(l_{2}-p_{2}\right)^{2}-m^{2}}+\frac{1}{\left(l_{3}+p_{1}\right)^{2}-m^{2}} \frac{1}{\left(l_{1}-p_{2}\right)^{2}-m^{2}}\right. \\
& \left.+\frac{1}{\left(l_{3}+p_{1}\right)^{2}-m^{2}} \frac{1}{\left(l_{2}-p_{2}\right)^{2}-m^{2}}\right) .
\end{aligned}
$$

Using the approximate form of the two scalars one graviton vertex in (49) and $\left(l_{1}+p_{1}\right)^{2}-m^{2} \approx 2 m l_{1}^{0}$ and taking the residue $2 m l_{i}^{0}=i \epsilon$, since for the rest of the residues we get a zero contribution at order $\mathcal{O}\left(\epsilon^{0}\right)$, we get

$$
\sum_{i=a}^{c} T_{(i)}^{(2) \mu \nu}=\left.32 \pi^{2} G_{N}^{2} m^{3} \int \prod_{n=1}^{2} \frac{d^{d+1} l_{n}}{(2 \pi)^{2 d}} \frac{\tau_{(3) \alpha \beta, 00}^{\mu \nu}\left(l_{1}+q, q\right) \cdot \mathcal{P}_{\phi \chi}{ }^{\alpha \beta} \cdot \tau_{(3) 00,00}^{\phi \chi}\left(-l_{2}, l_{1}+q\right)}{\left(\overrightarrow{l_{1}}\right)^{2}\left(\overrightarrow{l_{2}}\right)^{2}\left(\vec{l}_{3}\right)^{2}\left(\overrightarrow{l_{1}}+\vec{q}\right)^{2}}\right|_{l_{1}^{0}=l_{2}^{0}=0},
$$

with

$$
\begin{aligned}
\eta_{\mu \nu} \tau_{(3) \phi \chi, 00}^{\mu \nu}\left(l_{1}+q, q\right)= & \left(l^{\mu} l^{\nu}-(l+q)^{\mu}(l+q)^{\nu}-q^{\mu} q^{\nu}\right)-\frac{3}{2} \eta^{\mu \nu} \vec{q}^{2}\left(\eta^{\mu \nu}-(d-1) \delta_{0}^{\mu} \delta_{0}^{\nu}\right) \\
& +\frac{\eta^{\mu \nu}}{2}\left(\vec{l}_{1}^{2}-\left(\overrightarrow{l_{1}}+\vec{q}\right)^{2}\right)-\frac{5-d}{2} \delta_{0}^{\mu} \delta_{0}^{\nu}\left(\vec{l}_{1}^{2}+\left(\overrightarrow{l_{1}}+\vec{q}\right)^{2}\right),
\end{aligned}
$$


and

$$
\begin{aligned}
\delta_{\mu}^{0} \delta_{\nu}^{0} \tau_{(3) \phi \chi, 00}^{\mu \nu}\left(l_{1}+q, q\right)= & \frac{1}{d-1}\left((d-3)\left((l+q)^{\mu}(l+q)^{\nu}+q^{\mu} q^{\nu}\right)+(d-1)\left(l_{1}^{\mu} l_{1}^{\nu}-\frac{\vec{l}_{1}^{2}}{2}\left(3 \delta_{0}^{\mu} \delta_{0}^{\nu}-\eta^{\mu \nu}\right)\right)\right. \\
& \left.+\frac{\eta^{\mu \nu}-\delta_{0}^{\mu} \delta_{0}^{\nu}}{2}\left(\vec{q}^{2}(d-5)+(3 d-7)\left(\vec{l}_{1}+\vec{q}\right)^{2}\right)\right),
\end{aligned}
$$

and

$$
\begin{aligned}
\tau_{(3) 00,00}^{\mu \nu}(l, q)= & \frac{1}{d-1}\left((d-2)\left(l^{\mu} l^{\nu}+(l+q)^{\mu}(l+q)^{\nu}+q^{\mu} q^{\nu}+\frac{3}{2} \eta^{\mu \nu} \vec{q}^{2}\right)\right. \\
& \left.-2(d-2)\left(\vec{l}_{1}^{2}+\left(\overrightarrow{l_{1}}+\vec{q}\right)^{2}\right)\left(\delta_{0}^{\mu} \delta_{0}^{\mu}-\frac{\eta^{\mu \nu}}{4}\right)-2(d-3) \vec{q}^{2} \delta_{0}^{\mu} \delta_{0}^{\mu}\right) .
\end{aligned}
$$

Using the LiteRed code $[34,35]$ in $d$ dimensions, we find that all the contributions are proportional to the master integral as expected from the general discussion of Sec. II B

$$
\begin{aligned}
J_{(2)}(\vec{q}) & =\int \prod_{i=1}^{2} \frac{d^{d} \vec{l}_{i}}{(2 \pi)^{d}} \frac{\vec{q}^{2}}{\prod_{i=1}^{2} \vec{l}_{i}^{2}\left(\vec{l}_{1}+\vec{l}_{2}+\vec{q}\right)^{2}} \\
& =-\frac{\vec{q}^{2}}{32 \pi^{2}(d-3)}-\left(-3+\gamma_{E}-\log (4 \pi)+\log \left(\vec{q}^{2}\right)\right) \vec{q}^{2}+O(d-3),
\end{aligned}
$$

where $\gamma_{E}=0.57721 \ldots$ is the Euler-Mascheroni constant [38].

We find for the 00-component

$$
\sum_{i=a}^{c} T_{(i)}^{(2) 00}=\frac{32 \pi^{2} G_{N}^{2} m^{3}}{3} \frac{6 d^{3}-45 d^{2}+134 d-160}{(d-4)(d-1)^{2}} J_{(2)}\left(\vec{q}^{2}\right),
$$

and for the trace part

$$
\sum_{i=a}^{c} T_{(i)}^{(2) \mu \nu} \eta_{\mu \nu}=-\frac{32 \pi^{2} G_{N}^{2} m^{3}}{3} \frac{10 d^{3}-63 d^{2}+123 d-86}{(d-1)^{2}} J_{(2)}\left(\vec{q}^{2}\right) .
$$

\section{The diagrams $(d)$}

The diagram $(d)$ after symmetrization over the massive scalar legs reads

$$
\begin{aligned}
T_{(d)}^{(2) \mu \nu}= & -\frac{32 G_{N}^{2} \pi^{2}}{3 m} \int \prod_{n=1}^{3} \frac{d^{d+1} l_{n}}{(2 \pi)^{2 d}} \frac{\delta\left(l_{1}+l_{2}+l_{3}+q\right)}{l_{1}^{2} l_{2}^{2} l_{3}^{2}}\left(\frac{1}{\left(l_{1}+p_{1}\right)^{2}-m^{2}+i \epsilon} \frac{1}{\left(l_{2}-p_{2}\right)^{2}-m^{2}+i \epsilon}\right. \\
& \left.+\frac{1}{\left(l_{3}+p_{1}\right)^{2}-m^{2}+i \epsilon} \frac{1}{\left(l_{1}-p_{2}\right)^{2}-m^{2}+i \epsilon}+\frac{1}{\left(l_{3}+p_{1}\right)^{2}-m^{2}+i \epsilon} \overline{\left(l_{2}-p_{2}\right)^{2}-m^{2}+i \epsilon}\right) \\
& \times \tau^{\gamma \delta}\left(p_{1}, l_{1}+p_{1}\right) \tau^{\sigma \tau}\left(l_{1}+p_{1},-l_{2}+p_{1}\right) \tau^{l \theta}\left(l_{2}-p_{2},-p_{2}\right) \tau_{(4) \gamma \delta, \sigma \tau, l \theta}^{\mu \nu}\left(q, l_{1}, l_{2}, l_{3}\right),
\end{aligned}
$$

and leads to the contribution

$$
T_{(d)}^{(2) \mu \nu}=-\left.\frac{64 \pi^{2} G_{N}^{2} m^{3}}{3} \int \prod_{n=1}^{2} \frac{d^{d+1} l_{n}}{(2 \pi)^{d}} \frac{\tau_{(4) 00,00,00}^{\mu \nu}\left(q, l_{1}, l_{2},-l_{1}-l_{2}-q\right)}{\left(\overrightarrow{l_{1}}\right)^{2}\left(\vec{l}_{2}\right)^{2}\left(\vec{l}_{1}+\overrightarrow{l_{2}}+\vec{q}\right)^{2}}\right|_{l_{1}^{0}=l_{2}^{0}=0}
$$

with the vertex 


$$
\begin{aligned}
\tau_{(4) 00,00,00}^{\mu \nu}\left(q, l_{1}, l_{2}, l_{3}\right)= & \frac{1}{(d-1)^{2}}\left(\vec{q}^{2} \frac{\delta_{0}^{\mu} \delta_{0}^{\nu}}{2}\left(7 d^{2}-45 d+70\right)-\vec{q}^{2} \frac{\eta^{\mu \nu}}{2}(d-2)(6 d-23)\right. \\
& +(d-2)\left((9-2 d) q^{\mu} q^{\nu}+(7-2 d)\left(l_{1}^{\mu} l_{1}^{\nu}+l_{2}^{\mu} l_{2}^{\nu}+l_{3}^{\mu} l_{3}^{\nu}\right)\right) \\
& \left.+\frac{d-2}{2}\left(\vec{l}_{1}^{2}+\vec{l}_{2}^{2}+\vec{l}_{3}^{2}\right)\left(\delta_{0}^{\mu} \delta_{0}^{\nu}(7 d-23)-\eta^{\mu \nu}(2 d-9)\right)\right) .
\end{aligned}
$$

Evaluating these integral we find, for the 00-component

$$
T_{(d)}^{(2) 00}=-\frac{32 \pi^{2} G_{N}^{2} m^{3}}{3} \frac{(4-d)(6-d)}{(d-1)^{2}} J_{(2)}\left(\vec{q}^{2}\right),
$$

and for the trace part

$$
T_{(d)}^{(2) \mu \nu} \eta_{\mu \nu}=\frac{64 \pi^{2} G_{N}^{2} m^{3}}{3} \frac{3 d^{3}-20 d^{2}+41 d-30}{(d-1)^{2}} J_{(2)}\left(\vec{q}^{2}\right) .
$$

\section{The two-loop contribution to the metric components}

Summing up all the contributions the two-loop stresstensor is given by

$$
\begin{aligned}
\left\langle T_{\mu \nu}^{(2)}\right\rangle= & \pi^{2} G_{N}^{2} m^{3}\left(c_{1}^{(2)}(d) \delta_{\mu}^{0} \delta_{\nu}^{0}+c_{2}^{(2)}(d)\left(\frac{q_{\mu} q_{\nu}}{q^{2}}-\eta_{\mu \nu}\right)\right) \\
& \times J_{(2)}\left(q^{2}\right),
\end{aligned}
$$

with the coefficients given by

$$
\begin{aligned}
c_{1}^{(2)}(d)= & \frac{32}{3(d-4)(d-1)^{3}} \\
& \times\left(9 d^{4}-70 d^{3}+203 d^{2}-254 d+104\right), \\
c_{2}^{(2)}(d)= & \frac{64(d-2)}{3(d-4)(d-1)^{3}}\left(2 d^{3}-13 d^{2}+25 d-10\right),
\end{aligned}
$$

and the expression for the master integral

$$
J_{(2)}\left(\vec{q}^{2}\right)=\frac{\Gamma(3-d) \Gamma\left(\frac{d-2}{2}\right)^{3}}{(4 \pi)^{d} \Gamma\left(\frac{3(d-2)}{2}\right)}\left(\vec{q}^{2}\right)^{d-2} .
$$

From which we extract the metric components using the relations (39) [using the definition of $\rho(r, d)$ in (40)]

$$
\begin{aligned}
& h_{0}^{(3)}(r, d)=-\frac{8(3 d-7)(d-2)^{3}}{(d-4)(d-1)^{3}} \rho(r, d)^{3}, \\
& h_{1}^{(3)}(r, d)=\frac{8\left(7 d^{4}-63 d^{3}+214 d^{2}-334 d+212\right)}{3(d-3)(d-4)(d-1)^{3}} \rho(r, d)^{3}, \\
& h_{2}^{(3)}(r, d)=-\frac{8(d-2)^{2}\left(2 d^{3}-13 d^{2}+25 d-10\right)}{(d-3)(d-4)(d-1)^{3}} \rho(r, d)^{3} .
\end{aligned}
$$

\section{Three-loop amplitude}

The diagrams contributing to the classical corrections at third post-Minkowskian order of the metric at the two-loop graphs

$$
i \mathcal{M}_{3}^{(3)}\left(p_{1}, q\right)=-\sqrt{32 \pi G_{N}} T^{(3) \mu \nu} \epsilon_{\mu \nu},
$$

where the three-loop stress-tensor is given by five distinct diagrams

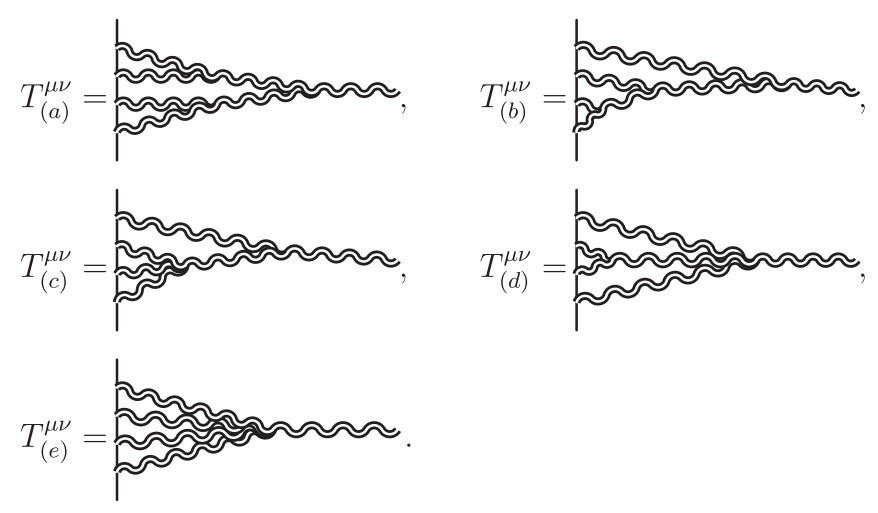

As before, we permute the internal momenta such that by taking the residue at $2 m l_{i}^{0}=i \epsilon$ from the massive propagators, we extract the nonanalytic terms which contribute to the classical metric in the static limit. After taking the residues and including the symmetry factors 


$$
\begin{aligned}
& T_{(a)}^{(3) \mu \nu}=\left.64 \pi^{3} G_{N}^{3} m^{4} \int \prod_{n=1}^{3} \frac{d^{d} \vec{l}_{n}}{(2 \pi)^{d}} \frac{\tau_{(3) \pi \rho, \sigma \tau}^{\mu \nu}\left(l_{1}+l_{2}, q\right) \tau_{(3)}^{\pi \rho}\left(-l_{1}, l_{1}+l_{2}\right) \tau_{(3)}^{\sigma \tau}\left(-l_{3}, l_{3}+l_{4}\right)}{\left(\vec{l}_{1}\right)^{2}\left(\vec{l}_{2}\right)^{2}\left(\vec{l}_{3}\right)^{2}\left(\vec{l}_{4}\right)^{2}\left(\vec{l}_{1}+\vec{l}_{2}\right)^{2}\left(\vec{l}_{3}+\vec{l}_{4}\right)^{2}}\right|_{l_{1}^{0}=l_{2}^{0}=l_{3}^{0}=0}, \\
& T_{(b)}^{(3) \mu \nu}=\left.256 \pi^{3} G_{N}^{3} m^{4} \int \prod_{n=1}^{3} \frac{d^{d} \vec{l}_{n}}{(2 \pi)^{d}} \frac{\tau_{(3) \sigma \tau, 00}^{\mu \nu}\left(l_{1}+q, q\right) \tau_{(3)}^{\pi \rho}\left(-l_{3}, l_{3}+l_{4}\right) \tau_{(3) 00, \pi \rho}^{\sigma \tau}\left(-l_{2}, l_{1}+q\right)}{\left(\vec{l}_{1}\right)^{2}\left(\vec{l}_{2}\right)^{2}\left(\vec{l}_{3}\right)^{2}\left(\vec{l}_{4}\right)^{2}\left(\vec{l}_{1}+\vec{q}\right)^{2}\left(\vec{l}_{3}+\vec{l}_{4}\right)^{2}}\right|_{l_{1}^{0}=l_{2}^{0}=l_{3}^{0}=0}, \\
& T_{(c)}^{(3) \mu \nu}=-\left.\frac{512 \pi^{3} G_{N}^{3} m^{4}}{3} \int \prod_{n=1}^{3} \frac{d^{d} \vec{l}_{n}}{(2 \pi)^{d}} \frac{\tau_{(3) \alpha \beta, 00}^{\mu \nu}\left(l_{1}+q, q\right) \tau_{(4) 00,00,00}^{\alpha \beta}\left(l_{1}+q, l_{2}, l_{3}, l_{4}\right)}{\left(\vec{l}_{1}\right)^{2}\left(\vec{l}_{2}\right)^{2}\left(\vec{l}_{3}\right)^{2}\left(\vec{l}_{4}\right)^{2}\left(\vec{l}_{1}+\vec{q}\right)^{2}}\right|_{l_{1}^{0}=l_{2}^{0}=l_{3}^{0}=0}, \\
& T_{(d) \mu \nu}^{(3) \mu \nu}=-256 \pi^{3} G_{N}^{3} m^{4} \int \prod_{n=1}^{3} \frac{d^{d} \vec{l}_{n}^{0}}{(2 \pi)^{d}} \frac{\tau_{(3)}^{\gamma \delta}\left(-l_{3}^{0}, l_{3}+l_{4}\right) \tau_{(4) \gamma \delta, 00,00}^{\mu \nu}\left(q, l_{1}, l_{2}, l_{3}+l_{4}\right)}{\left(\vec{l}_{1}\right)^{2}\left(\vec{l}_{2}\right)^{2}\left(\vec{l}_{3}\right)^{2}\left(\vec{l}_{4}\right)^{2}\left(\vec{l}_{3}+\vec{l}_{4}\right)^{2}} \\
& T_{(e) \mu \nu}^{(3) \mu \nu}=\left.\frac{256 \pi^{3} G_{N}^{3} m^{4}}{3} \int \prod_{n=1}^{3} \frac{d^{d} \vec{l}_{n}}{(2 \pi)^{d}} \frac{\tau_{(5) 00,00,00,00}^{\mu \nu}\left(q, l_{1}, l_{2}, l_{3}, l_{4}\right)}{\left(\vec{l}_{1}\right)^{2}\left(\vec{l}_{2}\right)^{2}\left(\vec{l}_{3}\right)^{2}\left(\vec{l}_{4}\right)^{2}}\right|_{l_{1}^{0}=l_{2}^{0}=l_{3}^{0}=0},
\end{aligned}
$$

with the five-graviton vertex contribution

$$
\begin{aligned}
& \tau_{(5) 00,00,00,00}^{\mu \nu}\left(k_{1}, k_{2}, k_{3}, k_{4}, k_{5}\right) \\
& :=\tilde{\tau}_{(5) \alpha \beta, \gamma \delta, \epsilon \eta, \kappa \lambda}^{\mu \nu}\left(k_{1}, k_{2}, k_{3}, k_{4}, k_{5}\right) P_{00}^{\alpha \beta} P_{00}^{\gamma \delta} P_{00}^{\epsilon \eta} P_{00}^{\kappa \lambda} \\
& =\frac{1}{4(d-1)^{3}}\left(4 \delta_{\mu}^{0} \delta_{\nu}^{0}\left(4\left(2 d^{3}-18 d^{2}+57 d-61\right) k_{1}^{2}+(d-2)\left(8 d^{2}-47 d+79\right) \sum_{i=2}^{5} k_{i}^{2}\right)\right. \\
& \quad-(d-2) \eta_{\mu \nu}\left(\left(29 d^{2}-191 d+362\right) k_{1}^{2}+\left(7 d^{2}-61 d+142\right) \sum_{i=2}^{5} k_{i}^{2}\right) \\
& \left.\quad+2(d-2)\left(\left(11 d^{2}-73 d+150\right) k_{1 \mu} k_{1 \nu}+\left(7 d^{2}-53 d+102\right)\left(k_{2 \mu} k_{2 \nu}+k_{3 \mu} k_{3 \nu}+k_{4 \mu} k_{4 \nu}+k_{5 \mu} k_{5 \nu}\right)\right)\right)
\end{aligned}
$$

where the vertex $\tau_{(5) \alpha \beta, \gamma \delta, \epsilon \eta, \kappa \lambda}^{\mu \nu}\left(k_{1}, k_{2}, k_{3}, k_{4}, k_{5}\right)$ has been derived using the results of [39].

The integral reduction is done using the LiteRed code [34,35] in $d$ dimensions. In agreement with the general analysis of Sec. II B, we find that the classical contribution is proportional to the single master integral

$$
J_{(3)}\left(\vec{q}^{2}\right)=\int \frac{d^{d} \vec{l}_{1} d^{d} \vec{l}_{2} d^{d} \vec{l}_{3}}{(2 \pi)^{3 d}} \frac{\vec{q}^{2}}{\vec{l}_{1}^{2} \vec{l}_{2}^{2} \vec{l}_{3}^{2}\left(\vec{l}_{1}+\vec{l}_{2}+\vec{l}_{3}+\vec{q}\right)^{2}} \text {. }
$$

\section{The $\mu=\nu=0$ component}

$$
\begin{aligned}
& T_{(a)}^{(3) 00}=-\frac{32 \pi^{3} G_{N}^{3} m^{4}}{3} \frac{3 d^{5}-169 d^{4}+1378 d^{3}-4592 d^{2}+7256 d-4752}{(d-4)^{2}(d-1)^{3}} J_{(3)}\left(\vec{q}^{2}\right), \\
& T_{(b)}^{(3) 00}=-\frac{128 \pi^{3} G_{N}^{3} m^{4}}{3} \frac{68 d^{6}-1003 d^{5}+6211 d^{4}-20820 d^{3}+40020 d^{2}-41584 d+17824}{(d-4)(d-3)(3 d-4)(d-1)^{3}} J_{(3)}\left(\vec{q}^{2}\right), \\
& T_{(c)}^{(3) 00}=\frac{64 \pi^{3} G_{N}^{3} m^{4}}{3} \frac{37 d^{5}-502 d^{4}+2731 d^{3}-7486 d^{2}+10164 d-5256}{(d-3)(3 d-4)(d-1)^{3}} J_{(3)}\left(\vec{q}^{2}\right), \\
& T_{(d)}^{(3) 00}=\frac{32 \pi^{3} G_{N}^{3} m^{4}}{3} \frac{53 d^{4}-615 d^{3}+2690 d^{2}-5572 d+4840}{(d-4)(d-1)^{3}} J_{(3)}\left(\vec{q}^{2}\right), \\
& T_{(e)}^{(3) 00}=64 \pi^{3} G_{N}^{3} m^{4} \frac{(6-d)\left(d^{2}-7 d+14\right)}{(d-1)^{3}} J_{(3)}\left(\vec{q}^{2}\right) .
\end{aligned}
$$




\section{Contraction with $\eta_{\mu \nu}$}

$$
\begin{aligned}
T_{(a)}^{(3) \mu \nu} \eta_{\mu \nu} & =\frac{32 \pi^{3} G_{N}^{3} m^{4}}{3} \frac{85 d^{6}-1126 d^{5}+6307 d^{4}-19114 d^{3}+32944 d^{2}-30472 d+11952}{(d-4)^{2}(d-1)^{3}} J_{(3)}\left(\vec{q}^{2}\right), \\
T_{(b)}^{(3) \mu \nu} \eta_{\mu \nu} & =\frac{128 \pi^{3} G_{N}^{3} m^{4}}{3} \frac{168 d^{6}-2231 d^{5}+12319 d^{4}-35796 d^{3}+57396 d^{2}-48304 d+16736}{(d-4)(3 d-4)(d-1)^{3}} J_{(3)}\left(\vec{q}^{2}\right), \\
T_{(c)}^{(3) \mu \nu} \eta_{\mu \nu} & =-\frac{64 \pi^{3} G_{N}^{3} m^{4}}{3} \frac{147 d^{6}-1801 d^{5}+8727 d^{4}-21555 d^{3}+28942 d^{2}-20148 d+5688}{(3 d-4)(d-1)^{4}} J_{(3)}\left(\vec{q}^{2}\right), \\
T_{(d)}^{(3) \mu \nu} \eta_{\mu \nu} & =-\frac{32 \pi^{3} G_{N}^{3} m^{4}}{3} \frac{179 d^{5}-2146 d^{4}+10305 d^{3}-24614 d^{2}+28972 d-13704}{(d-4)(d-1)^{3}} J_{(3)}\left(\vec{q}^{2}\right), \\
T_{(e)}^{(3) \mu \nu} \eta_{\mu \nu} & =\frac{64 \pi^{3} G_{N}^{3} m^{4}}{3} \frac{29 d^{4}-274 d^{3}+973 d^{2}-1484 d+852}{(d-1)^{3}} J_{(3)}\left(\vec{q}^{2}\right) .
\end{aligned}
$$

\section{The classical three-loop contribution to the stress-tensor}

Summing up all the contributions we get for the three-loop stress-tensor

$$
\left\langle T_{\mu \nu}^{(3)}\right\rangle=\pi^{3} G_{N}^{3} m^{4}\left(c_{1}^{(3)}(d) \delta_{\mu}^{0} \delta_{\nu}^{0}+c_{2}^{(3)}(d)\left(\frac{q_{\mu} q_{\nu}}{q^{2}}-\eta_{\mu \nu}\right)\right) J_{(3)}\left(q^{2}\right),
$$

with the master integral

$$
J_{(3)}\left(q^{2}\right)=\frac{\Gamma\left(\frac{8-3 d}{2}\right) \Gamma\left(\frac{d-2}{2}\right)^{4}}{8^{d} \pi^{\frac{3 d}{2}} \Gamma(2(d-2))}|\vec{q}|^{3(d-2)},
$$

and the three-loop coefficients are given by

$$
\begin{aligned}
c_{1}^{(3)}(d)= & -\frac{64}{3(d-3)(d-4)^{2}(d-1)^{4}} \times\left(56 d^{7}-889 d^{6}+5868 d^{5}\right. \\
& \left.-20907 d^{4}+43434 d^{3}-52498 d^{2}+33888 d-8760\right), \\
c_{2}^{(3)}(d)= & -\frac{64}{3(d-3)(d-4)^{2}(d-1)^{4}} \times\left(45 d^{7}-670 d^{6}+4167 d^{5}\right. \\
& \left.-14016 d^{4}+27430 d^{3}-30916 d^{2}+18104 d-3952\right) .
\end{aligned}
$$

Using the relations (39) we obtained the three-loop contribution to the metric from the classical stress-tensor in (126) [using the notation for $\rho$ in (40)]

$$
\begin{aligned}
& h_{0}^{(4)}(r, d)=\frac{16(d-2)^{3}\left(14 d^{3}-85 d^{2}+165 d-106\right)}{3(d-3)(d-4)(d-1)^{4}} \rho(r, d)^{4}, \\
& h_{1}^{(4)}(r, d)=-\frac{8\left(39 d^{7}-691 d^{6}+5155 d^{5}-21077 d^{4}+51216 d^{3}-74346 d^{2}+60168 d-21208\right)}{3(d-3)(d-4)^{2}(d-1)^{4}(3 d-8)} \rho(r, d)^{4}, \\
& h_{2}^{(4)}(r, d)=\frac{16(d-2)^{2}\left(45 d^{6}-580 d^{5}+3007 d^{4}-8002 d^{3}+11426 d^{2}-8064 d+1976\right)}{3(d-3)(d-4)^{2}(d-1)^{4}(3 d-8)} \rho(r, d)^{4} .
\end{aligned}
$$

\section{NONMINIMAL COUPLINGS AND RENORMALIZED METRIC}

The stress-tensor and the metric components have ultraviolet divergences. These divergences can be removed by the addition of the nonminimal couplings made from the powers of the covariant derivative $\nabla_{\mu}$ acting on a single power of the Riemann tensor and its contractions. The Bianchi identity on the Riemann tensor $\nabla_{\mu} R_{\nu \rho \sigma \lambda}+\nabla_{\nu} R_{\rho \mu \sigma \lambda}+\nabla_{\rho} R_{\mu \nu \sigma \lambda}=0$, implies that 


$$
\nabla_{\mu} R_{\rho \sigma \lambda}^{\mu}=\nabla_{\sigma} R_{\rho \lambda}-\nabla_{\lambda} R_{\rho \sigma}, \quad \nabla_{\mu} R_{\nu}^{\mu}=\frac{1}{2} \nabla_{\nu} R .
$$

The counter-terms are powers of covariant derivative acting on a single power of the Ricci tensor and Ricci scalar. Therefore the counterterms are given by the following nonminimal couplings

$$
\begin{aligned}
\delta^{(n)} S^{\mathrm{ct}}= & \left(G_{N} m\right)^{\frac{2 n}{d-2}} \int d^{d+1} x \sqrt{-g}\left(\alpha^{(n)}(d)\left(\nabla^{2}\right)^{n-1} R \partial_{\mu} \phi \partial^{\mu} \phi\right. \\
& +\left(\beta_{0}^{(n)}(d) \nabla_{\mu} \nabla_{\nu}\left(\nabla^{2}\right)^{n-2} R\right. \\
& \left.\left.+\beta_{1}^{(n)}(d)\left(\nabla^{2}\right)^{n-1} R_{\mu \nu}\right) \partial^{\mu} \phi \partial^{\nu} \phi\right) .
\end{aligned}
$$

where $\alpha^{(n)}(d), \beta_{0}^{(n)}(d)$ and $\beta_{1}^{(n)}(d)$ are dimensionless coefficients depending on the space-time dimension. The power of $G_{N} m$ is determined by dimensional analysis, and give the correct order of $G_{N}$ in all dimensions. The first nonminimal coupling with $n=1$ is given by

$$
\begin{aligned}
\delta^{(1)} S^{\mathrm{ct}}= & \left(G_{N} m\right)^{\frac{2}{d-2}} \int d^{d+1} x \sqrt{-g}\left(\alpha^{(1)}(d) R \partial_{\mu} \phi \partial^{\mu} \phi\right. \\
& \left.+\beta^{(1)}(d) R^{\mu \nu} \partial_{\mu} \phi \partial_{\nu} \phi\right) .
\end{aligned}
$$

This non-minimal coupling has been introduced in [27] in four dimensions and [24] in five dimensions. We will see that up to three-loop order the renormalization of the static metric component only require the counter-term $\alpha^{(1)} R \partial_{\mu} \phi \partial^{\mu} \phi$, whereas both couplings are needed for the cancellation of the stress-tensor divergences. This coupling is induced by harmonic gauge condition $[20,24]$ and the value of its coefficient depends on the choice of gauge. In our gauge, the de Donder gauge, this corresponds to $\alpha=0$ in the work of [24] and $\xi=\frac{1}{4}$ in the work of [20]. Since we are working in fixed gauge we will not discuss further the gauge dependence of the higher-order nonminimal coupling coefficients, but we expect that the gauge dependence of these coefficients will be an extension of the discussion in [[24] Appendix B].

The power of the Newton constant in (89) is an integer only in four dimensions with $d=3$ and five dimensions $d=4$. Therefore this counterterm will not appear in dimensions $D \geq 6$.

In four dimensions, from five-loop order, or the sixth post-Minkowskian order $O\left(G_{N}^{6}\right)$, one expects that higher derivative nonminimal couplings will be needed to get finite stress-tensor components. In dimensions five and six, the higher-derivative nonminimal couplings arise at lower loop order.

In five dimensions one needs to consider higher-derivative nonminimal couplings $\delta^{(n)} S^{\text {ct }}$ with $n \geq 2$ for removing the divergences in the stress-tensor. The nonminimal coupling at this order is then given by

$$
\begin{aligned}
\delta^{(2)} S^{\mathrm{ct}}= & \left(G_{N} m\right)^{\frac{4}{d-2}} \int d^{d+1} x \sqrt{-g}\left(\alpha^{(2)}(d) \square R \partial_{\mu} \phi \partial^{\mu} \phi\right. \\
& \left.+\left(\beta_{0}^{(2)}(d) \nabla_{\mu} \nabla_{\nu} R+\beta_{1}^{(2)}(d) \square R_{\mu \nu}\right) \partial^{\mu} \phi \partial^{\nu} \phi\right) .
\end{aligned}
$$

We will need the nonminimal coupling

$$
\begin{aligned}
\delta^{(3)} S^{\mathrm{ct}}= & \left(G_{N} m\right)^{\frac{6}{d-2}} \int d^{d+1} x \sqrt{-g}\left(\alpha^{(3)}(d)\left(\nabla^{2}\right)^{2} R \partial_{\mu} \phi \partial^{\mu} \phi\right. \\
& \left.+\left(\beta_{0}^{(3)}(d) \nabla_{\mu} \nabla_{\nu} \nabla^{2} R+\beta_{1}^{(3)}(d)\left(\nabla^{2}\right)^{2} R_{\mu \nu}\right) \partial^{\mu} \phi \partial^{\nu} \phi\right),
\end{aligned}
$$

for removing the two-loop divergence in the stress-tensor in six $(d=5)$ dimensions and the three-loop divergence in five $(d=4)$ dimensions. In five dimensions $(d=4)$ the metric, up to $G_{N}^{4}$, is renormalized using only the $n=1$ and the metric is finite to all order in six dimensions $(d=5)$.

The higher-order non-minimal couplings $\delta^{(n)} S^{\text {ct }}$ with $n \geq 2$ will not contribute to the classical limit when inserted into graphs with loops, because they contribute to higher powers in the momentum transfer $\vec{q}$, and are subleading with respect to the classical contributions. Their tree-level insertions will contribute to the renormalization of the stress-tensor but thanks to the properties of the Fourier transform they will not contribute to the metric components.

\section{A. Tree-level insertions}

We give the contribution of the insertions of the nonminimal counterterms with $n=1$ in (89), with $n=2$ in (90) and with $n=3$ in (91) in the tree-level graph.

\section{Insertion of $\delta^{(1)} S^{\mathrm{ct}}$}

The insertion of the nonminimal couplings $\delta^{(1)} S^{\text {ct }}$ in (89) into the tree-level diagram



leads to the stress-tensor contribution in $d+1$ dimensions

$$
\begin{aligned}
\delta^{(1)}\left\langle T_{\mu \nu}^{(0)}\right\rangle= & -\vec{q}^{2}\left(G_{N} m\right)^{\frac{2}{d-2} m} \\
& \times\left(-\beta^{(1)}(d) \delta_{\mu}^{0} \delta_{\nu}^{0}+2 \alpha^{(1)}(d)\left(\frac{q_{\mu} q_{\nu}}{q^{2}}-\eta_{\mu \nu}\right)\right),
\end{aligned}
$$


and using (7) this contributes to the metric components

$$
\begin{gathered}
\delta^{(1)} h_{0}^{(1)}(r, d)=0, \\
\delta^{(1)} h_{1}^{(1)}(r, d)=\frac{16 \alpha^{(1)}(d) \Gamma\left(\frac{d}{2}\right)}{\pi^{\frac{d-2}{2}}}\left(\frac{\left(G_{N} m\right)^{\frac{1}{d-2}}}{r}\right)^{d}, \\
\delta^{(1)} h_{2}^{(1)}(r, d)=-\frac{32 \alpha^{(1)}(d) \Gamma\left(\frac{d+2}{2}\right)}{\pi^{\frac{d-2}{2}}}\left(\frac{\left(G_{N} m\right)^{\frac{1}{d-2}}}{r}\right)^{d} .
\end{gathered}
$$

Thanks to the properties of the Fourier transformation (see Appendix A) only the coefficient $\alpha(d)$ contributes to static metric perturbation.

\section{Insertion of $\delta^{(2)} S^{\mathrm{ct}}$}

The insertion of the nonminimal couplings $\delta^{(2)} S^{\mathrm{ct}}$ in (90) into the tree-level diagram

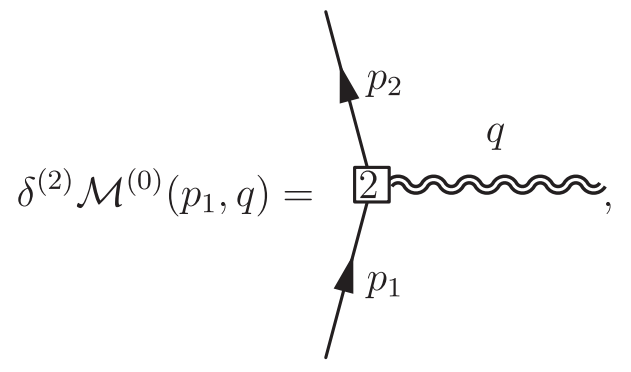

leads to the stress-tensor condition in $d+1$ dimensions

$$
\begin{aligned}
\delta^{(2)}\left\langle T_{\mu \nu}^{(0)}\right\rangle= & |\vec{q}|^{4}\left(G_{N} m\right)^{\frac{4}{d-2}} m\left(-\beta_{1}^{(2)}(d) \delta_{\mu}^{0} \delta_{\nu}^{0}\right. \\
& \left.+2\left(\alpha^{(2)}(d)+\frac{1}{2} \beta_{0}^{(2)}(d)\right)\left(\frac{q_{\mu} q_{\nu}}{q^{2}}-\eta_{\mu \nu}\right)\right) .
\end{aligned}
$$

Because of the vanishing of the Fourier transforms

$$
\int_{\mathbb{R}^{d}}|\vec{q}|^{2} e^{i \vec{q} \cdot \vec{x}} \frac{d^{d} \vec{q}}{(2 \pi)^{d}}=0, \quad \int_{\mathbb{R}^{d}} \frac{q_{i} q_{j}}{|\vec{q}|^{2}}|\vec{q}|^{2} e^{i \vec{q} \cdot \vec{x}} \frac{d^{d} \vec{q}}{(2 \pi)^{d}}=0,
$$

this extra contribution to the stress-tensor does not affect the metric components

$$
\begin{gathered}
\delta^{(2)} h_{0}^{(1)}(r, d)=0, \\
\delta^{(2)} h_{1}^{(1)}(r, d)=0, \\
\delta^{(2)} h_{2}^{(1)}(r, d)=0 .
\end{gathered}
$$

3. Insertion of $\delta^{(3)} S^{\text {ct }}$

The insertion of the nonminimal couplings $\delta^{(3)} S^{\mathrm{ct}}$ in (91) into the tree-level diagram

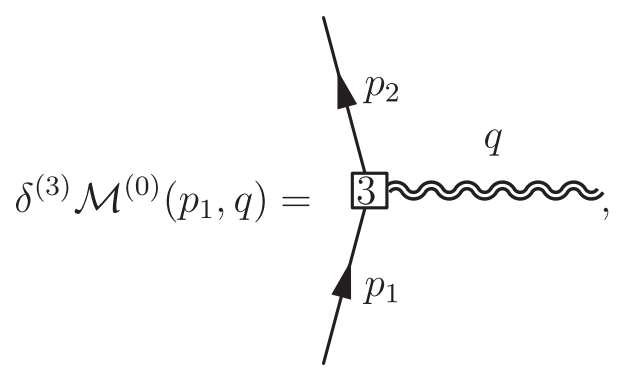

leads to the stress-tensor condition in six dimensions $(d=5)$

$$
\begin{aligned}
\delta^{(3)}\left\langle T_{\mu \nu}^{(0)}\right\rangle= & -|\vec{q}|^{6}\left(G_{N} m\right)^{\frac{6}{d-2}} m\left(-\beta_{1}^{(3)}(d) \delta_{\mu}^{0} \delta_{\nu}^{0}\right. \\
& \left.+2\left(\alpha^{(3)}(d)+\frac{1}{4} \beta_{0}^{(3)}(d)\right)\left(\frac{q_{\mu} q_{\nu}}{q^{2}}-\eta_{\mu \nu}\right)\right) .
\end{aligned}
$$

Because of the vanishing of the Fourier transforms

$$
\int_{\mathbb{R}^{d}}|\vec{q}|^{4} e^{i \vec{q} \cdot \vec{x}} \frac{d^{d} \vec{q}}{(2 \pi)^{d}}=0, \quad \int_{\mathbb{R}^{d}} \frac{q_{i} q_{j}}{|\vec{q}|^{2}}|\vec{q}|^{4} e^{i \vec{q} \cdot \vec{x}} \frac{d^{d} \vec{q}}{(2 \pi)^{d}}=0,
$$

this extra contribution to the stress-tensor does not affect the metric components

$$
\delta^{(2)} h_{0}^{(1)}(r, d)=0
$$

$$
\begin{aligned}
& \delta^{(2)} h_{1}^{(1)}(r, d)=0, \\
& \delta^{(2)} h_{2}^{(1)}(r, d)=0 .
\end{aligned}
$$

\section{B. One-loop insertions}

We give the contribution of the insertions of the counterterms (88) with $n=1$ in (89) in the one-loop graph.

\section{Insertion of $\delta^{(1)} S^{\mathrm{ct}}$}

The insertion of the nonminimal coupling in (89) in the one-loop graph 


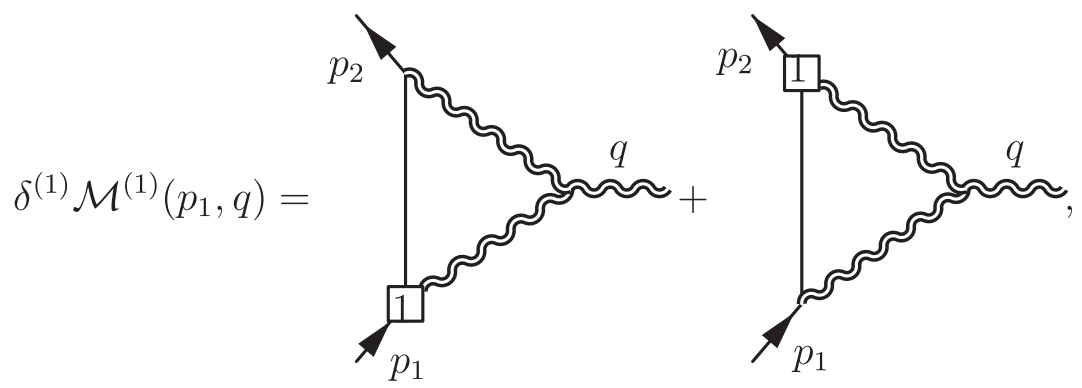

leads to the stress-tensor contribution

$$
\begin{aligned}
\delta^{(1)}\left\langle T_{\mu \nu}^{(1)}\right\rangle & =32 i \alpha^{(1)}(d) \pi\left(G_{N} m\right)^{\frac{d}{d-2}} m^{2} \int \frac{d^{d} \vec{l}}{(2 \pi)^{d}} \frac{\tau_{10 \alpha \beta, \gamma \delta}^{\mu \nu}(l, q) \mathcal{P}_{00}^{\alpha \beta} l^{\gamma} l^{\delta}}{l^{2}(l+q)^{2}}\left[\frac{1}{\left(l+p_{1}\right)^{2}-m^{2}+i \epsilon}+\frac{1}{\left(l-p_{2}\right)^{2}-m^{2}+i \epsilon}\right] \\
& =8 \pi \alpha^{(1)}(d)\left(G_{N} m\right)^{\frac{d}{d-2}} m \vec{q}^{2} \frac{d-2}{(d-1)^{2}}\left(d \delta_{\mu}^{0} \delta_{\nu}^{0}+\frac{q_{\mu} q_{\nu}}{q^{2}}-\eta_{\mu \nu}\right) J_{(1)}\left(\vec{q}^{2}\right) .
\end{aligned}
$$

where we used that

$$
\eta_{\mu \nu} \tau_{1000, \gamma \delta}^{\mu \nu}(l, q) l^{\gamma} l^{\delta}=\frac{\vec{l}_{1}^{2}}{2}\left(\vec{q}^{2}+\left(\vec{l}_{1}+\vec{q}\right)^{2}-\vec{l}_{1}^{2}\right),
$$

and

$$
\begin{aligned}
\delta_{\mu}^{0} \delta_{\nu}^{0} \tau_{1000, \gamma \delta}^{\mu \nu}(l, q) l^{\gamma} l^{\delta}= & \frac{1}{2(d-1)}\left((d-2) \vec{q}^{4}+(d-2)\left(\overrightarrow{l_{1}}+\vec{q}\right)^{2}\left(\left(\vec{l}_{1}+\vec{q}\right)^{2}-2 \vec{q}^{2}\right)-\vec{l}_{1}^{4}\right. \\
& \left.-(d-3) \vec{l}_{1}^{2}\left(\left(\overrightarrow{l_{1}}+\vec{q}\right)^{2}+\vec{q}^{2}\right)\right) .
\end{aligned}
$$

Using the Fourier transforms

$$
\begin{gathered}
\int_{\mathbb{R}^{d}} J_{(1)}\left(\vec{q}^{2}\right) e^{i \vec{q} \cdot \vec{x}} \frac{d^{d} \vec{q}}{(2 \pi)^{d}}=-\frac{\Gamma\left(\frac{d}{2}\right)^{2}}{2 \pi^{d} r^{2(d-1)}}, \\
\int_{\mathbb{R}^{d}} \frac{q_{i} q_{j}}{\vec{q}^{2}} J_{(1)}\left(\vec{q}^{2}\right) e^{i \vec{q} \cdot \vec{x}} \frac{d^{d} \vec{q}}{(2 \pi)^{d}}=\frac{\Gamma\left(\frac{d-2}{2}\right) \Gamma\left(\frac{d}{2}\right)}{4 \pi^{d} r^{2(d-1)}}\left(\delta_{i j}-2(d-1) \frac{x_{i} x_{j}}{r}\right) .
\end{gathered}
$$

and the relation between the stress-tensor and the metric components in (7) we obtain the following contribution to the metric components

$$
\begin{aligned}
& \delta^{(1)} h_{0}^{(2)}(r, d)=64 \alpha^{(1)}(d) \frac{(d-2) \Gamma\left(\frac{d}{2}\right)^{2}}{(d-1) \pi^{d-2}}\left(\frac{\left(G_{N} m\right)^{\frac{1}{d-2}}}{r}\right)^{2(d-1)}, \\
& \delta^{(1)} h_{1}^{(2)}(r, d)=-64 \alpha^{(1)}(d) \frac{\Gamma\left(\frac{d}{2}\right)^{2}}{(d-1) \pi^{d-2}}\left(\frac{\left(G_{N} m\right)^{\frac{1}{d-2}}}{r}\right)^{2(d-1)}, \\
& \delta^{(1)} h_{2}^{(2)}(r, d)=128 \alpha^{(1)}(d) \frac{\Gamma\left(\frac{d}{2}\right)^{2}}{(d-1) \pi^{d-2}}\left(\frac{\left(G_{N} m\right)^{\frac{1}{d-2}}}{r}\right)^{2(d-1)} .
\end{aligned}
$$




\section{Two insertions of $\delta^{(1)} S^{\text {ct }}$}

Two insertions of the nonminimal coupling $\delta^{(1)} S^{\text {ct }}$ in (89) in the one-loop graph

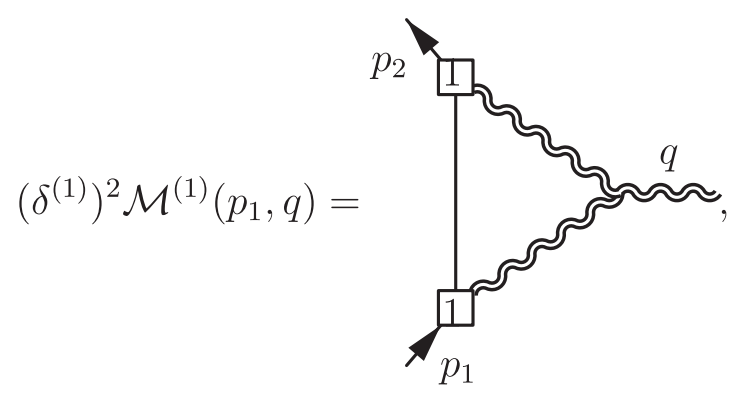

leads to the stress-tensor contribution

$$
\begin{aligned}
\left(\delta^{1}\right)^{2}\left\langle T_{\mu \nu}^{(1)}\right\rangle= & \frac{2\left(\alpha^{(1)}(d)\right)^{2}\left(G_{N} m\right)^{\frac{d+2}{d-2}} \pi m \vec{q}^{4}}{d-1}\left(\delta_{\mu}^{0} \delta_{\nu}^{0}-(d-2)\right. \\
& \left.\times\left(\frac{q_{\mu} q_{\nu}}{q^{2}}-\eta_{\mu \nu}\right)\right) J_{(1)}\left(\vec{q}^{2}\right),
\end{aligned}
$$

and the metric contributions

$$
\begin{aligned}
& \left(\delta^{1}\right)^{2} h_{0}^{(2)}(r, d)=0, \\
& \left(\delta^{1}\right)^{2} h_{1}^{(2)}(r, d)=\frac{64\left(\alpha^{(1)}(d)\right)^{2}}{\pi^{d-2}} \Gamma\left(\frac{d}{2}\right)^{2}\left(\frac{\left(G_{N} m\right)^{\frac{1}{d-2}}}{r}\right)^{2 d}, \\
& \left(\delta^{1}\right)^{2} h_{2}^{(2)}=\frac{64 d(d-2)\left(\alpha^{(1)}(d)\right)^{2}}{\pi^{d-2}} \Gamma\left(\frac{d}{2}\right)^{2}\left(\frac{\left(G_{N} m\right)^{\frac{1}{d-2}}}{r}\right)^{2 d} .
\end{aligned}
$$

\section{Two-loop insertions}

For the insertion of the nonminimal coupling $\delta^{(1)} S^{\mathrm{ct}}$ in (89) in the two-loop graph one needs to sum over all the contributions in Table I. The classical limit of the sum of all these graphs lead to the following contribution to the stresstensor

$$
\begin{aligned}
\delta^{(1)}\left\langle T_{\mu \nu}^{(2)}\right\rangle= & -\frac{128 \pi^{2}(d-2) \alpha^{(1)}(d)}{3(d-4)(3 d-4)(d-1)^{2}}\left(G_{N} m\right)^{\frac{2(d-1)}{d-2}} m \vec{q}^{2}\left(\left(3 d^{3}-19 d^{2}+28 d-10\right) \delta_{\mu}^{0} \delta_{\nu}^{0}\right. \\
& \left.+\left(3 d^{3}-15 d^{2}+18 d-4\right)\left(\frac{q_{\mu} q_{\nu}}{q^{2}}-\eta_{\mu \nu}\right)\right) J_{(2)}\left(\vec{q}^{2}\right),
\end{aligned}
$$

which leads to the following contributions to the metric components

TABLE I. Insertion of the non-minimal coupling in the two-loop graph.

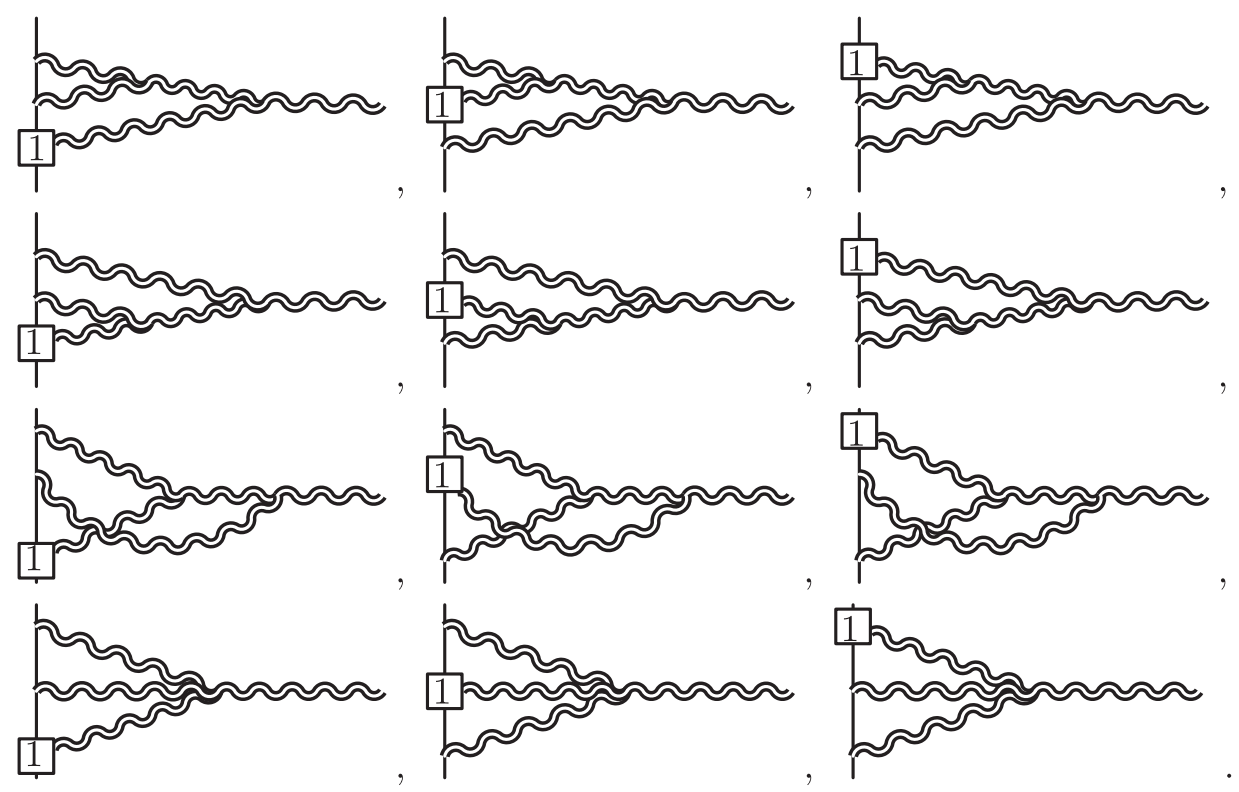




$$
\begin{aligned}
& \delta^{(1)} h_{0}^{(3)}(r, d)=-\frac{512 \alpha^{(1)}(d)}{d-1} \frac{\Gamma\left(\frac{d}{2}\right)^{3}}{\pi^{\frac{3}{2}(d-2)}}\left(\frac{\left(G_{N} m\right)^{\frac{1}{d-2}}}{r}\right)^{3 d-4}, \\
& \delta^{(1)} h_{1}^{(3)}(r, d)=\frac{256 \alpha^{(1)}(d)\left(3 d^{3}-23 d^{2}+46 d-28\right)}{(d-4)(d-2)(d-1)^{2}(3 d-4)} \frac{\Gamma\left(\frac{d}{2}\right)^{3}}{\pi^{\frac{3}{2}(d-2)}}\left(\frac{\left(G_{N} m\right)^{\frac{1}{d-2}}}{r}\right)^{3 d-4}, \\
& \delta^{(1)} h_{2}^{(3)}(r, d)=-\frac{256 \alpha^{(1)}(d)\left(3 d^{3}-15 d^{2}+18 d-4\right)}{(d-4)(d-2)(d-1)^{2}} \frac{\Gamma\left(\frac{d}{2}\right)^{3}}{\pi^{\frac{3}{2}(d-2)}}\left(\frac{\left(G_{N} m\right)^{\frac{1}{d-2}}}{r}\right)^{3 d-4} .
\end{aligned}
$$

\section{The renormalized metric in four dimensions}

The metric components have ultraviolet poles in four dimensions from two-loop order. We show how the addition of the nonminimal couplings leads to finite renormalized metric components.

\section{The two-loop renormalization}

The two-loop metric components in (76) have a divergence in four dimensions $(d=3)$

$$
\begin{aligned}
& h_{0}^{(3)}(r, d)=O(1), \\
& h_{1}^{(3)}(r, d)=-\frac{2}{3(d-3)}\left(\frac{G_{N} m}{r}\right)^{3}+O(1), \\
& h_{2}^{(3)}(r, d)=\frac{2}{d-3}\left(\frac{G_{N} m}{r}\right)^{3}+O(1) .
\end{aligned}
$$

This divergence is cancelled by adding the metric contribution from the non-minimal coupling in (94)

$h_{i}^{\text {renor (3) }}(r, d):=h_{i}^{(3)}(r, d)+\delta^{(1)} h_{i}^{(1)}(r, d), \quad i=0,1,2$

and setting the $\alpha^{(1)}(d)$ coefficient to be

$\alpha^{(1)}(d)=\frac{1}{12(d-3)}+a^{(1)}(3)-\frac{\log (2)}{6}+O(d-3)$.

The resulting renormalized two-loop metric reads

$$
\begin{aligned}
h_{0}^{\text {renor }(3)}(r, d)= & 2\left(\frac{G_{N} m}{r}\right)^{3}+O(d-3), \\
h_{1}^{\text {renor }(3)}(r, d)= & \frac{4}{3}\left(-\frac{1}{2}+6 a^{(1)}(3)\right. \\
& \left.+\log \left(\frac{r C_{E}}{G_{N} m}\right)\right)\left(\frac{G_{N} m}{r}\right)^{3}+O(d-3), \\
h_{2}^{\text {renor }(3)}(r, d)= & 4\left(\frac{1}{3}-6 a^{(1)}(3)-\log \left(\frac{r C_{E}}{G_{N} m}\right)\right)\left(\frac{G_{N} m}{r}\right)^{3} \\
& +O(d-3) .
\end{aligned}
$$

where we have introduced the following combination of the Euler-Mascheroni constant [38] and $\pi$

$$
C_{E}:=\sqrt{\pi} e^{\frac{\gamma_{E}}{2}}
$$

The divergence in the two-loop stress-tensor in (73)

$$
\left\langle T_{\mu \nu}^{(2)}\right\rangle=\frac{G_{N}^{2} \vec{q}^{2} m^{3}}{6(d-3)}\left(2 \delta_{\mu}^{0} \delta_{\nu}^{0}+\left(\frac{q_{\mu} q_{\nu}}{q^{2}}-\eta_{\mu \nu}\right)\right)+O(1),
$$

is canceled by adding the contribution in (93) from the nonminimal coupling with the following choice of $\beta^{(1)}(d)$ coefficient

$$
\beta^{(1)}(d)=-\frac{1}{3(d-3)}+O(1)
$$

Notice that this computation does not determine the finite part of the $\alpha^{(1)}(d)$ and $\beta^{(1)}(d)$. They are free scales in the logarithms. We will show in Sec. VI that this freedom is totally reabsorbed in the change of coordinate and the Schwarzschild-Tangherlini metric does not have any ambiguity.

\section{The three-loop renormalization}

The three-loop metric components in (86) have a divergence in four dimensions $(d=3)$ given by

$$
\begin{aligned}
& h_{0}^{(4)}(r, d)=-\frac{2}{3(d-3)}\left(\frac{G_{N} m}{r}\right)^{4}+O(1), \\
& h_{1}^{(4)}(r, d)=\frac{2}{3(d-3)}\left(\frac{G_{N} m}{r}\right)^{4}+O(1), \\
& h_{2}^{(4)}(r, d)=-\frac{4}{3(d-3)}\left(\frac{G_{N} m}{r}\right)^{4}+O(1), .
\end{aligned}
$$

Adding to this contribution the (111) from the insertion of the nonminimal couplings at one-loop, and using the value of $\alpha^{(1)}(d)$ determined in (119), we obtain the renormalized three-loop metric 


$$
\begin{aligned}
h_{0}^{\text {renorm }(4)}(r)= & \left(-\frac{32}{3}+8 a^{(1)}(3)+\frac{4}{3} \log \left(\frac{r C_{E}}{G_{N} m}\right)\right)\left(\frac{G_{N} m}{r}\right)^{4} \\
& +O(d-3), \\
h_{1}^{\text {renorm }(4)}(r)= & \left(10-8 a^{(1)}(3)-\frac{4}{3} \log \left(\frac{r C_{E}}{G_{N} m}\right)\right)\left(\frac{G_{N} m}{r}\right)^{4} \\
& +O(d-3), \\
h_{2}^{\text {renorm }(4)}(r)= & \left(-\frac{86}{3}+16 a^{(1)}(3)+\frac{8}{3} \log \left(\frac{r C_{E}}{G_{N} m}\right)\right)\left(\frac{G_{N} m}{r}\right)^{4} \\
& +O(d-3) .
\end{aligned}
$$

The classical three-loop contribution to the stress-tensor has an ultraviolet divergence

$$
\begin{aligned}
\left\langle T_{\mu \nu}^{(3)}(\vec{q})\right\rangle= & -\frac{\pi G_{N}^{3} m^{4}|\vec{q}|^{\frac{3}{2}}}{48(d-3)}\left(3 \delta_{\mu}^{0} \delta_{\nu}^{0}+\left(\frac{q_{\mu} q_{\nu}}{q^{2}}-\eta_{\mu \nu}\right)\right) \\
& +O(1),
\end{aligned}
$$

this divergence is canceled by the addition of the contribution in (107) from the nonminimal coupling and the choice of $\alpha^{(1)}(d)$ in (119).

\section{E. The renormalized metric in five dimensions}

The metric components have ultraviolet divergences in five dimensions from one-loop order. We show how the addition of the nonminimal couplings leads to finite renormalized metric components.

\section{The one-loop renormalization}

The metric components in (58) have a divergence in five dimension $(d=4)$ given by

$$
\begin{aligned}
& h_{0}^{(2)}(r, d)=O(1), \\
& h_{1}^{(2)}(r, d)=-\frac{40}{9(d-4)}\left(\frac{G_{N} m}{\pi r^{2}}\right)^{2}+O(1), \\
& h_{2}^{(2)}(r, d)=\frac{160}{9(d-4)}\left(\frac{G_{N} m}{\pi r^{2}}\right)^{2}+O(1) .
\end{aligned}
$$

The divergences in the metric components (127) are canceled for the choice

$$
\alpha^{(1)}(d)=\frac{5}{18 \pi(d-4)}+a^{(1)}(5)+O(d-4),
$$

so that the renormalized metric components

$$
h_{i}^{\text {renor }(2)}(r, d):=h_{i}^{(2)}(r, d)+\delta^{(1)} h_{i}^{(1)}(r, d), \quad i=0,1,2,
$$

have a finite expansion near $d=4$

$$
\begin{aligned}
h_{0}^{\text {renor }(2)}(r, d)= & \frac{32}{9}\left(\frac{G_{N} m}{\pi r^{2}}\right)^{2}+O(d-4), \\
h_{1}^{\text {renor }(2)}(r, d)= & \frac{20}{9}\left(\frac{14}{15}+\frac{36 a^{(1)}(5) \pi}{5}+\log \left(\frac{r^{2} C_{E}^{2}}{G_{N} m}\right)\right) \\
& \times\left(\frac{G_{N} m}{\pi r^{2}}\right)^{2}+O(d-4), \\
h_{2}^{\text {renor }(2)}(r, d)= & -\frac{80}{9}\left(\frac{7}{30}+\frac{36 a^{(1)}(5) \pi}{5}+\log \left(\frac{r^{2} C_{E}^{2}}{G_{N} m}\right)\right) \\
& \times\left(\frac{G_{N} m}{\pi r^{2}}\right)^{2}+O(d-4) .
\end{aligned}
$$

where $C_{E}$ is defined in (121).

Thanks to the properties of the Fourier transform, only the coefficient $\alpha^{(1)}(d)$ enters the counterterm contribution to the metric component. To determine as well the coefficient $\beta^{(1)}(d)$ in (89) one needs to look at the divergences of the stress-tensor

$$
\left\langle T_{\mu \nu}^{(1)}\right\rangle=\frac{G_{N} m^{2} \vec{q}^{2}}{18 \pi(d-4)}\left(7 \delta_{\mu}^{0} \delta_{\nu}^{0}+10\left(\frac{q_{\mu} q_{\nu}}{q^{2}}-\eta_{\mu \nu}\right)\right)+O(1)
$$

The cancellation of the pole fixes the pole part of $\beta^{(1)}(d)$ near five dimensions

$$
\beta^{(1)}(d)=-\frac{7}{18 \pi(d-4)}+O(1)
$$

\section{The two-loop renormalization}

The two-loop metric components in (76) have a divergence in five dimensions $(d=4)$

$$
\begin{aligned}
& h_{0}^{(3)}(r, d)=-\frac{320}{27(d-4)}\left(\frac{G_{N} m}{\pi r^{2}}\right)^{3}+O(1), \\
& h_{1}^{(3)}(r, d)=\frac{160}{27(d-4)}\left(\frac{G_{N} m}{\pi r^{2}}\right)^{3}+O(1), \\
& h_{2}^{(3)}(r, d)=-\frac{320}{27(d-4)}\left(\frac{G_{N} m}{\pi r^{2}}\right)^{3}+O(1) .
\end{aligned}
$$

The divergences in the metric components (76) are cancelled for the choice made at one-loop in (128), so that the renormalized metric components

$h_{i}^{\text {renor(3) }}(r, d):=h_{i}^{(3)}(r, d)+\delta^{(1)} h_{i}^{(2)}(r, d), \quad i=0,1,2$,

have a finite expansion near $d=4$ 


$$
\begin{aligned}
& h_{0}^{\text {renor }(3)}(r, d)=\frac{160}{27}\left(\frac{2}{15}+\frac{36 a^{(1)}(5) \pi}{5}+\log \left(\frac{r^{2} C_{E}^{2}}{G_{N} m}\right)\right)\left(\frac{G_{N} m}{\pi r^{2}}\right)^{3}+O(d-4), \\
& h_{1}^{\text {renor }(3)}(r, d)=-\frac{80}{27}\left(\frac{7}{15}+\frac{36 a^{(1)}(5) \pi}{5}+\log \left(\frac{r^{2} C_{E}^{2}}{G_{N} m}\right)\right)\left(\frac{G_{N} m}{\pi r^{2}}\right)^{3}+O(d-4), \\
& h_{2}^{\text {renor }(3)}(r, d)=\frac{160}{27}\left(-\frac{1}{15}+\frac{36 a^{(1)}(5) \pi}{5}+\log \left(\frac{r^{2} C_{E}^{2}}{G_{N} m}\right)\right)\left(\frac{G_{N} m}{\pi r^{2}}\right)^{3}+O(d-4) .
\end{aligned}
$$

The two-loop stress-tensor in (73) is not finite in $d=4$ as it diverges like

$$
\begin{aligned}
\left\langle T_{\mu \nu}^{(2)}\right\rangle= & \frac{5 G_{N}^{2} m^{3}|\vec{q}|^{4}}{162 \pi^{2}(d-4)^{2}}\left(4 \delta_{\mu}^{0} \delta_{\nu}^{0}+\frac{q_{\mu} q_{\nu}}{q^{2}}-\eta_{\mu \nu}\right)+\frac{5 G^{2} m^{3}|\vec{q}|^{4}}{162 \pi^{2}(d-4)}\left(\left(4 \log \left(\frac{\vec{q}^{2}}{4 \pi}\right)+4 \gamma_{E}-\frac{183}{20}\right) \delta_{\mu}^{0} \delta_{\nu}^{0}\right. \\
& \left.+\left(\log \left(\frac{\vec{q}^{2}}{4 \pi}\right)+\gamma_{E}-\frac{41}{20}\right)\left(\frac{q_{\mu} q_{\nu}}{q^{2}}-\eta_{\mu \nu}\right)\right)+O(1) .
\end{aligned}
$$

The addition of the counterterm in (107) from the nonminimal couplings in (89) is not enough for making the stress-tensor finite in $d=4$

$$
\begin{aligned}
\left\langle T_{\mu \nu}^{(2)}\right\rangle+\delta^{(1)}\left\langle T_{\mu \nu}^{(1)}\right\rangle= & -\frac{5 G_{N}^{2} m^{3}|\vec{q}|^{4}}{162 \pi^{2}(d-4)^{2}}\left(4 \delta_{\mu}^{0} \delta_{\nu}^{0}+\frac{q_{\mu} q_{\nu}}{q^{2}}-\eta_{\mu \nu}\right) \\
& +\frac{5 G^{2} m^{3}|\vec{q}|^{4}}{162 \pi^{2}(d-4)}\left(\left(4 \log \left(G_{N} m\right)-\frac{144 \pi a^{(1)}(5)}{5}-\frac{109}{60}\right) \delta_{\mu}^{0} \delta_{\nu}^{0}\right. \\
& \left.+\left(\left(\log \left(G_{N} m\right)+\frac{17}{60}-\frac{36}{5} \pi a^{(1)}(5)\right)\left(\frac{q_{\mu} q_{\nu}}{q^{2}}-\eta_{\mu \nu}\right)\right)\right)+O(1) .
\end{aligned}
$$

We need to consider the addition of the counterterm from the insertion of $\delta^{(2)} S^{\mathrm{ct}}$ evaluated in Sec. IV A 2 with the values of the coefficient near $d=4$

$$
\begin{aligned}
\beta_{1}^{(2)}(d) & =\frac{1}{\pi^{2}}\left(\frac{10}{81(d-4)^{2}}+\frac{109+1728 \pi a^{(1)}(5)}{1944(d-4)}+a^{(2)}(5)+O(d-4)\right), \\
\alpha^{(2)}(d)+\frac{1}{2} \beta_{0}^{(2)}(d) & =-\frac{1}{2 \pi^{2}}\left(\frac{5}{162(d-4)^{2}}+\frac{432 \pi a^{(1)}(5)-17}{1944(d-4)}+b^{(2)}(5)+O(d-4)\right),
\end{aligned}
$$

plugged in (97) cancel the divergences in (137)

$$
\left\langle T_{\mu \nu}^{(2)}\right\rangle+\delta^{(1)}\left\langle T_{\mu \nu}^{(1)}\right\rangle+\delta^{(2)}\left\langle T_{\mu \nu}^{(0)}\right\rangle=O(1)
$$

\section{The three-loop renormalization}

The three-loop metric components in (86) have a divergence in five dimensions $(d=4)$

$$
\begin{aligned}
& h_{0}^{(4)}(r, d)=\frac{1280}{27(d-4)}\left(\frac{G_{N} m}{\pi r^{2}}\right)^{4}+O(1), \\
& h_{1}^{(4)}(r, d)=\left(\frac{400}{81(d-4)^{2}}-\frac{20\left(101+120 \log \left(r^{2} C_{E}^{2}\right)\right)}{243(d-4)}\right)\left(\frac{G_{N} m}{\pi r^{2}}\right)^{4}+O(1), \\
& h_{2}^{(4)}(r, d)=\left(\frac{3200}{81(d-4)^{2}}+\frac{160\left(187-120 \log \left(r^{2} C_{E}^{2}\right)\right)}{243}\right)\left(\frac{G_{N} m}{\pi r^{2}}\right)^{4}+O(1) .
\end{aligned}
$$

The divergences in the metric components (86) are cancelled for the choice made at one-loop in (128), so that the renormalized metric components 


$$
h_{i}^{\text {renor }(4)}(r, d):=h_{i}^{(4)}(r, d)+\delta^{(1)} h_{i}^{(3)}(r, d)+\left(\delta^{1}\right)^{2} h_{i}^{(2)}(r, d), \quad i=0,1,2
$$

have a finite expansion near $d=4$

$$
\begin{aligned}
h_{0}^{\text {renor }(4)}(r, d)= & -\frac{128}{243}\left(23+324 a^{(1)}(5) \pi+45 \log \left(\frac{r^{2} C_{E}^{2}}{G_{N} m}\right)\right)\left(\frac{G_{N} m}{\pi r^{2}}\right)^{4}+O(d-4), \\
h_{1}^{\text {renor }(4)}(r, d)= & \frac{100}{81}\left(\left(\frac{36 a^{(1)}(5) \pi}{5}+\log \left(\frac{r^{2} C_{E}^{2}}{\left.G_{N} m\right)}\right)\left(\frac{161}{30}+\frac{36}{5} a^{(1)}(5) \pi+\log \left(\frac{\left(r^{2} C_{E}^{2}\right.}{\left.G_{N} m\right)}\right)\right.\right.\right. \\
& \left.+\frac{7085}{1800}\right) \times\left(\frac{G_{N} m}{\pi r^{2}}\right)^{4}+O(d-4), \\
h_{2}^{\text {renor }(4)}(r, d)= & -\frac{800}{81}\left(\left(\frac{36 a^{(1)}(5) \pi}{5}+\log \left(\frac{r^{2} C_{E}^{2}}{G_{N} m}\right)\right)\left(\frac{41}{15}-\frac{36}{5} a^{(1)}(5) \pi-\log \left(\frac{r^{2} C_{E}^{2}}{G_{N} m}\right)\right)\right. \\
& \left.+\frac{2381}{900}\right) \times\left(\frac{G_{N} m}{\pi r^{2}}\right)^{4}+O(d-4) .
\end{aligned}
$$

The three-loop stress-tensor in (83) is not finite in $d=4$ as it diverges like

$$
\begin{aligned}
\left\langle T_{\mu \nu}^{(3)}\right\rangle= & \frac{25 G_{N}^{3} m^{4}|\vec{q}|^{6}}{5832 \pi^{3}(d-4)^{3}}\left(-\frac{1}{2} \delta_{\mu}^{0} \delta_{\nu}^{0}+\frac{q_{\mu} q_{\nu}}{q^{2}}-\eta_{\mu \nu}\right) \\
& +\frac{25 G_{N}^{3} m^{4}|\vec{q}|^{6}}{3888 \pi^{3}(d-4)^{2}}\left(-\frac{1}{2}\left(\log \left(\frac{\vec{q}^{2}}{4 \pi}\right)+\gamma_{E}-\frac{41}{6}\right) \delta_{\mu}^{0} \delta_{\nu}^{0}\right. \\
& \left.+\left(\log \left(\frac{\vec{q}^{2}}{4 \pi}\right)+\gamma_{E}-\frac{17}{10}\right)\left(\frac{q_{\mu} q_{\nu}}{q^{2}}-\eta_{\mu \nu}\right)\right) \\
& +\frac{225 G_{N}^{3} m^{4}|\vec{q}|^{6}}{839808 \pi^{3}(d-4)}\left(\frac{1}{2}\left(\frac{70939}{450}+\pi^{2}-18\left(\log \left(\frac{\vec{q}^{2}}{4 \pi}\right)+\gamma_{E}-\frac{41}{6}\right)^{2}\right) \delta_{\mu}^{0} \delta_{\nu}^{0}\right. \\
& \left.+\left(\frac{4769}{450}-\pi^{2}+18\left(\log \left(\frac{\vec{q}^{2}}{4 \pi}\right)+\gamma_{E}-\frac{17}{10}\right)^{2}\right)\left(\frac{q_{\mu} q_{\nu}}{q^{2}}-\eta_{\mu \nu}\right)\right)+O(1) .
\end{aligned}
$$

The addition of the counterterms in $\left(\delta^{1}\right)^{2}\left\langle T_{\mu \nu}^{(1)}\right\rangle$ in (113), and $\delta^{(1)}\left\langle T_{\mu \nu}^{(2)}\right\rangle$ in (115) from the non-minimal couplings in (89) is not enough for making the stress-tensor finite in $d=4$

$$
\begin{aligned}
\left\langle T_{\mu \nu}^{(3)}\right\rangle+\left(\delta^{1}\right)^{2}\left\langle T_{\mu \nu}^{(1)}\right\rangle+\delta^{(1)}\left\langle T_{\mu \nu}^{(2)}\right\rangle \\
=\frac{25 G_{N}^{3} m^{4}|\vec{q}|^{6}}{5832 \pi^{3}(d-4)^{3}}\left(-\frac{1}{2} \delta_{\mu}^{0} \delta_{\nu}^{0}+\frac{q_{\mu} q_{\nu}}{q^{2}}-\eta_{\mu \nu}\right) \\
\quad+\frac{25 G_{N}^{3} m^{4}|\vec{q}|^{6}}{3888 \pi^{3}(d-4)^{2}}\left(-\frac{1}{2}\left(\frac{25}{12}+\frac{36}{5} a^{(1)}(5) \pi-\log \left(G_{N} m\right)\right) \delta_{\mu}^{0} \delta_{\nu}^{0}\right. \\
\left.\quad+\left(\frac{1}{60}+\frac{36}{5} a^{(1)}(5) \pi-\log \left(G_{N} m\right)\right)\left(\frac{q_{\mu} q_{\nu}}{q^{2}}-\eta_{\mu \nu}\right)\right)-\frac{25 G_{N}^{3} m^{4}|\vec{q}|^{6}}{5184 \pi^{3}(d-4)} \\
\quad \times\left(\left(\frac{27487}{48600}+a^{(1)}(5) \pi\left(1+\frac{288}{25} a^{(1)}(5) \pi\right)-\frac{\log \left(G_{N} m\right)}{3}\left(\frac{7}{2}+\log \left(G_{N} m\right)+\frac{72}{5} a^{(1)}(5) \pi\right)\right) \delta_{\mu}^{0} \delta_{\nu}^{0}\right. \\
\left.\quad+\left(\frac{6749}{16200}-\frac{6 a^{(1)}(5) \pi}{25}\left(1+144 a^{(1)}(5) \pi\right)-\log \left(G_{N} m\right)\left(\frac{19}{30}+\log \left(G_{N} m\right)-\frac{72}{5} a^{(1)}(5) \pi\right)\right)\left(\frac{q_{\mu} q_{\nu}}{q^{2}}-\eta_{\mu \nu}\right)\right) \\
\quad+O(1) .
\end{aligned}
$$

We need to consider the addition of the counterterm from the insertion of $\delta^{(3)} S^{\text {ct }}$ evaluated in Sec. IV A 3 with the values of the coefficient near $d=4$ 


$$
\begin{aligned}
\beta_{1}^{(3)}(d)= & \frac{25}{11664 \pi^{3}(d-4)^{3}}+\frac{5\left(432 \pi a^{(1)}(5)+125\right)}{93312 \pi^{3}(d-4)^{2}} \\
& +\frac{559872\left(\pi a^{(1)}(5)\right)^{2}+486000 \pi a^{(1)}(5)+27487}{6718464 \pi^{3}(d-4)}+O(1), \\
\alpha^{(3)}(d)+\frac{1}{4} \beta_{0}^{(3)}(d)= & \frac{25}{11664 \pi^{3}(d-4)^{3}}+\frac{2160 \pi a^{(1)}(5)+5}{93312 \pi^{3}(d-4)^{2}} \\
& +\frac{559872\left(\pi a^{(1)}(5)\right)^{2}+3888 \pi a^{(1)}(5)-6749}{6718464 \pi^{3}(d-4)}+O(1),
\end{aligned}
$$

plugged in (97) cancel the divergences in (137)

$\left\langle T_{\mu \nu}^{(2)}\right\rangle+\delta^{(1)}\left\langle T_{\mu \nu}^{(2)}\right\rangle+\left(\delta^{(1)}\left\langle T_{\mu \nu}^{(2)}\right\rangle+\delta^{(3)}\left\langle T_{\mu \nu}^{(0)}\right\rangle=O(1)\right.$.

\section{F. The renormalized stress-tensor in six dimensions}

In six dimensions, the metric component are finite to all order in perturbation but the two-loop stress-tensor in (73) presents an ultraviolet divergence in six dimensions $(d=5)$

$$
\begin{aligned}
\left\langle T_{\mu \nu}^{(2)}\right\rangle= & -\frac{G_{N}^{2} m^{3}|\vec{q}|^{6}}{40320 \pi^{2}(d-5)}\left(49 \delta_{\mu}^{0} \delta_{\nu}^{0}+15\left(\frac{q_{\mu} q_{\nu}}{q^{2}}-\eta_{\mu \nu}\right)\right) \\
& +O(1),
\end{aligned}
$$

which is canceled by the addition of the insertion of the nonminimal coupling $\delta^{(3)} S^{\text {ct }}$ at tree-level in (102) with the choice of the coefficients

$$
\begin{aligned}
\alpha^{(3)}(d)+\frac{1}{4} \beta_{0}^{(3)}(d) & =-\frac{15}{80640 \pi^{2}(d-5)}+O(1), \\
\beta_{1}^{(3)}(d) & =-\frac{49}{40320 \pi^{2}(d-5)}+O(1) .
\end{aligned}
$$

\section{THE SCHWARZSCHILD-TANGHERLINI METRIC IN de DONDER GAUGE IN FOUR, FIVE, AND SIX DIMENSIONS}

The Schwarzschild-Tangherlini [40] space-time metric in $d+1$ dimensions is given by the Tangherlini solution, using $\rho(r, d)$ defined in $(40){ }^{7}$

\footnotetext{
${ }^{7}$ In spherical coordinate the metric reads

$$
d s^{2}=\left(1-\frac{\mu}{r^{d-2}}\right) d t^{2}-\frac{d r^{2}}{1-\frac{\mu}{r^{d-1}}}-r^{2} d \Omega_{d-1}
$$
with $\mu=\frac{16 \pi G_{N} m}{(d-1) \Omega_{d-1}}$ and $\Omega_{d-1}=\frac{2 \pi^{\frac{d}{2}}}{\Gamma\left(\frac{d}{2}\right)}$ is the area of the unit $(d-1)$ -
sphere.
}

$$
\begin{aligned}
d s_{\mathrm{Schw}}^{2}= & \left(1-4 \frac{d-2}{d-1} \rho(r, d)\right) d t^{2}-d \vec{x}^{2} \\
& -\frac{4 \frac{d-2}{d-1} \rho(r, d)}{1-4 \frac{d-2}{d-1} \rho(r, d)} \frac{(\vec{x} \cdot d \vec{x})^{2}}{r^{2}} .
\end{aligned}
$$

As explained in Sec. II the amplitude computation selects the de Donder gauge in (5). We make the coordinate transformation $(t, \vec{x}) \rightarrow(t, f(r) \vec{x})$ so that the Schwarzschild metric reads

$$
d s^{2}=h_{0}(r) d t^{2}-h_{1}(r) d \vec{x}^{2}-h_{2}(r) \frac{(\vec{x} \cdot d \vec{x})^{2}}{r^{2}}
$$

with $r=|\vec{x}|$ and

$h_{0}(r):=1-4 \frac{d-2}{d-1} \frac{\rho(r, d)}{f(r)^{d-2}}$,

$h_{1}(r):=f(r)^{2}$,

$h_{2}(r):=-f(r)^{2}-f(r)^{d-2} \frac{\left(f(r)+r \frac{d f(r)}{d r}\right)^{2}}{f(r)^{d-2}-4 \frac{d-2}{d-1} \rho(r, d)}$.

The de Donder gauge condition (5) then reads

$2(d-1) h_{2}(r)=r \frac{d}{d r}\left(h_{0}(r)+(d-2) h_{1}(r)-h_{2}(r)\right)$.

We will be solving the de Donder gauge condition (5) in four dimensions $(d=3)$, five dimensions $(d=4)$, and six dimensions $(d=5)$, using the post-Minkowskian expansion

$$
f(r)=1+\sum_{n \geq 1} f_{n}(r) \rho(r, d)^{n}
$$

with the condition at each order that

$$
\lim _{r \rightarrow+\infty} f_{n}(r) / r^{n}=0 .
$$




\section{A. The metric in the de Donder gauge in four dimensions}

The de Donder gauge condition (5) in $d=3$ reads

$$
4 h_{2}(r)=r \frac{d}{d r}\left(h_{0}(r)+h_{1}(r)-h_{2}(r)\right),
$$

supplemented with the asymptotic boundary condition

$$
\lim _{r \rightarrow \infty} f(r)=1
$$

This differential equation implies either that $f(r)=$ $C / r$, which does not satisfy the boundary condition (157), or $f(r)$ satisfies the differential equation, with $x=G_{N} m / r$

$$
\begin{aligned}
& x f(x)^{3}(2 x-f(x)) \frac{d^{2} f(x)}{d x^{2}}+(x f(x))^{2}\left(\frac{d f(x)}{d x}\right)^{2} \\
& \quad+2 f(x)^{3}(f(x)-3 x) \frac{d f(x)}{d x}-3(f(x))^{4}+8(f(x))^{3} x+(f(x))^{2}-4 f(x) x+4 x^{2}=0 .
\end{aligned}
$$

We solve the Eq. (158) using a series expansion in $G_{N} m$ using (154) and the boundary condition (155). The result to the order $\left(G_{N} m\right)^{7}$ is given by

$$
\begin{aligned}
f(r)= & 1+\frac{G_{N} m}{r}+2\left(\frac{G_{N} m}{r}\right)^{2}+\frac{2}{3} \log \left(\frac{r C_{3}}{G_{N} m}\right)\left(\frac{G_{N} m}{r}\right)^{3} \\
& +\left(\frac{2}{3}-\frac{4}{3} \log \left(\frac{r C_{3}}{G_{N} m}\right)\right)\left(\frac{G_{N} m}{r}\right)^{4}+\left(-\frac{21}{25}+\frac{32}{15} \log \left(\frac{r C_{3}}{G_{N} m}\right)\right)\left(\frac{G_{N} m}{r}\right)^{5} \\
& +\left(\frac{112}{75}-\frac{28}{15} \log \left(\frac{r C_{3}}{G_{N} m}\right)\left(\frac{G_{N} m}{r}\right)^{6}\right. \\
& +\left(\frac{50023}{34300}+\frac{1139}{2205} \log \left(\frac{r C_{3}}{G_{N} m}\right)+\frac{2}{7} \log \left(\frac{r C_{3}}{G_{N} m}\right)^{2}\right)\left(\frac{G_{N} m}{r}\right)^{7}+O\left(G_{N}^{8}\right) .
\end{aligned}
$$

This solution is finite and has $\log (r)$ terms from the order $G_{N}^{3}$. The solution has a single constant of integration $C_{3}$ associated with the scale of the logarithm.

\section{The metric perturbation}

In $d=3$ we derive components of the metric in perturbation by plugging the expression for $f(r)$ in (159) in (152). We obtain for the time component

$$
\begin{aligned}
h_{0}^{\mathrm{dD}}(r)= & 1-2 \frac{G_{N} m}{r}+2\left(\frac{G_{N} m}{r}\right)^{2}+2\left(\frac{G_{N} m}{r}\right)^{3}+\left(\frac{4}{3} \log \left(\frac{r C_{3}}{G_{N} m}\right)-6\right)\left(\frac{G_{N} m}{r}\right)^{4} \\
& +\left(-\frac{16}{3} \log \left(\frac{r C_{3}}{G_{N} m}\right)+\frac{10}{3}\right)\left(\frac{G_{N} m}{r}\right)^{5}+\left(\frac{124}{15} \log \left(\frac{r C_{3}}{G_{N} m}\right)+\frac{424}{75}\right)\left(\frac{G_{N} m}{r}\right)^{6} \\
& +\left(-\frac{8}{9} \log \left(\frac{r C_{3}}{G_{N} m}\right)^{2}+\frac{16}{15} \log \left(\frac{r C_{3}}{G_{N} m}\right)-\frac{674}{75}\right)\left(\frac{G_{N} m}{r}\right)^{7}+O\left(G_{N}^{8}\right),
\end{aligned}
$$

and for the spatial components

$$
\begin{aligned}
h_{1}^{\mathrm{dD}}(r)= & 1+2 \frac{G_{N} m}{r}+5\left(\frac{G_{N} m}{r}\right)^{2}+\left(\frac{4}{3} \log \left(\frac{r C_{3}}{G_{N} m}\right)+4\right)\left(\frac{G_{N} m}{r}\right)^{3} \\
& +\left(-\frac{4}{3} \log \left(\frac{r C_{3}}{G_{N} m}\right)+\frac{16}{3}\right)\left(\frac{G_{N} m}{r}\right)^{4}+\left(\frac{64}{15} \log \left(\frac{r C_{3}}{G_{N} m}\right)-\frac{26}{75}\right)\left(\frac{G_{N} m}{r}\right)^{5} \\
& +\left(\frac{4}{9} \log \left(\frac{r C_{3}}{G_{N} m}\right)^{2}-\frac{24}{5} \log \left(\frac{r C_{3}}{G_{N} m}\right)+\frac{298}{75}\right)\left(\frac{G_{N} m}{r}\right)^{6}+O\left(G_{N}^{7}\right),
\end{aligned}
$$

and 


$$
\begin{aligned}
h_{2}^{\mathrm{dD}}(r)= & -7\left(\frac{G_{N} m}{r}\right)^{2}-\left(4 \log \left(\frac{r C_{3}}{G_{N} m}\right)+\frac{38}{3}\right)\left(\frac{G_{N} m}{r}\right)^{3}+\left(\frac{8}{3} \log \left(\frac{r C_{3}}{G_{N} m}\right)-\frac{58}{3}\right)\left(\frac{G_{N} m}{r}\right)^{4} \\
& -\left(\frac{16}{3} \log \left(\frac{r C_{3}}{G_{N} m}\right)-\frac{32}{3}\right)\left(\frac{G_{N} m}{r}\right)^{5} \\
& +\left(\frac{4}{3} \log \left(\frac{r C_{3}}{G_{N} m}\right)^{2}+\frac{508}{45} \log \left(\frac{r C_{3}}{G_{N} m}\right)+\frac{7378}{225}\right)\left(\frac{G_{N} m}{r}\right)^{6}+O\left(G_{N}^{7}\right) .
\end{aligned}
$$

Notice the appearance of the $\log (r)^{2}$ at the sixth post-Minkowskian order, $G_{N}^{6}$, in the spatial components of the metric. This is one order less than the appearance in the time component. The same phenomenon happens for the $\log (r)$ contribution which appears one order earlier in the spatial component than in the time component.

\section{B. The metric in the de Donder gauge in five dimensions}

The de Donder gauge condition (5) in $d=4$ reads

$$
6 h_{2}(r)=r \frac{d}{d r}\left(h_{0}(r)+2 h_{1}(r)-h_{2}(r)\right)
$$

supplemented with the asymptotic boundary condition

$$
\lim _{r \rightarrow \infty} f(r)=1 .
$$

This differential equation implies either that $f(r)=C / r$, which does not satisfy the boundary condition (164), or $f(r)$ satisfies the differential equation, setting $x=G_{N} m /\left(\pi r^{2}\right)$

$$
\begin{aligned}
& x f(x)^{5}\left(8 x-3 f(x)^{2}\right) \frac{d^{2} f(x)}{d x^{2}}+8 f(x)^{4} x^{2}\left(\frac{d f(x)}{d x}\right)^{2}+f(x)^{5}\left(3 f(x)^{2}-16 x\right) \frac{d f(x)}{d x} \\
& -4 f(x)^{6}+(16 x+2) f(x)^{4}-\frac{32}{3} x f(x)^{2}+\frac{128 x^{2}}{9}=0 .
\end{aligned}
$$

We solve the equation (165) using a series expansion in $G_{N} m$ using (154) and the boundary condition (155). The result to the order $\left(G_{N} m\right)^{7}$ is given by

$$
\begin{aligned}
f(r)= & 1+\frac{2}{3} \frac{G_{N} m}{\pi r^{2}}+\frac{10}{9} \log \left(\frac{r^{2} C_{2}}{G_{N} m}\right)\left(\frac{G_{N} m}{\pi r^{2}}\right)^{2}-\frac{4}{81}\left(-8+45 \log \left(\frac{r^{2} C_{2}}{G_{N} m}\right)\right)\left(\frac{G_{N} m}{\pi r^{2}}\right)^{3} \\
& +\frac{67+3780 \log \left(\frac{r^{2} C_{2}}{G_{N} m}\right)\left(\frac{G_{N} m}{\pi r^{2}}\right)^{4}-\frac{32963+156420 \log \left(\frac{r^{2} C_{2}}{G_{N} m}\right)-43200 \log \left(\frac{r^{2} C_{2}}{G_{N} m}\right)^{2}}{21870}\left(\frac{G_{N} m}{\pi r^{2}}\right)^{5}}{131220}\left(\frac{409303+1620270 \log \left(\frac{r^{2} C_{2}}{G_{N} m}\right)-1087200 \log \left(\frac{r^{2} C_{2}}{G_{N} m}\right)^{2}}{\pi r^{2}}\right)^{6} \\
& +\frac{11148022313+37508666370 \log \left(\frac{r^{2} C_{2}}{G_{N} m}\right)-64367301600 \log \left(\frac{r^{2} C_{2}}{G_{N} m}\right)^{2}}{2362944150}\left(\frac{G_{N} m}{\pi r^{2}}\right)^{7} \\
& -\frac{4939200000}{2362944150} \log \left(\frac{r^{2} C_{2}}{G_{N} m}\right)^{3}\left(\frac{G_{N} m}{\pi r^{2}}\right)^{7}+O\left(G_{N}^{8}\right) .
\end{aligned}
$$

Again there is a single constant of integration $C_{2}$ arising as the scale of the $\log (r)$ arising from the $G_{N}^{2}$ order.

\section{The metric perturbation}

In $d=4$ we derive components of the metric in perturbation by plugging the expression for $f(r)$ in (166) in (152). 
We obtain for the time component

$$
\begin{aligned}
h_{0}^{\mathrm{dD}}(r)= & 1-\frac{8}{3} \frac{G_{N} m}{\pi r^{2}}+\frac{32}{9}\left(\frac{G_{N} m}{\pi r^{2}}\right)^{2}+\frac{32\left(-3+5 \log \left(\frac{r^{2} C_{2}}{G_{N} m}\right)\right)}{27}\left(\frac{G_{N} m}{\pi r^{2}}\right)^{3} \\
& -\frac{640\left(-2+9 \log \left(\frac{r^{2} C_{2}}{G_{N} m}\right)\right)}{243}\left(\frac{G_{N} m}{\pi r^{2}}\right)^{4}+O\left(G_{N}^{5}\right),
\end{aligned}
$$

and for the spatial components

$$
\begin{aligned}
h_{1}^{\mathrm{dD}}(r)= & 1+\frac{4}{3} \frac{G_{N} m}{\pi r^{2}}+\frac{4\left(1+5 \log \left(\frac{r^{2} C_{2}}{G_{N} m}\right)\right)}{9}\left(\frac{G_{N} m}{\pi r^{2}}\right)^{2}+\frac{\left(64-240 \log \left(\frac{r^{2} C_{2}}{G_{N} m}\right)\right)}{81}\left(\frac{G_{N} m}{\pi r^{2}}\right)^{3} \\
& +\frac{\left(323+2340 \log \left(\frac{r^{2} C_{2}}{G_{N} m}\right)+600 \log ^{2}\left(\frac{r^{2} C_{2}}{G_{N} m}\right)\right)}{486}\left(\frac{G_{N} m}{\pi r^{2}}\right)^{4}+O\left(G_{N}^{5}\right),
\end{aligned}
$$

and

$$
\begin{aligned}
h_{2}^{\mathrm{dD}}(r)= & \frac{40\left(1-2 \log \left(\frac{r^{2} C_{2}}{G_{N} m}\right)\right)}{9}\left(\frac{G_{N} m}{\pi r^{2}}\right)^{2}+\frac{32\left(-4+5 \log \left(\frac{r^{2} C_{2}}{G_{N} m}\right)\right)}{27}\left(\frac{G_{N} m}{\pi r^{2}}\right)^{3} \\
& +\frac{8\left(-31-1260 \log \left(\frac{r^{2} C_{2}}{G_{N} m}\right)+300 \log \left(\frac{r^{2} C_{2}}{G_{N} m}\right)^{2}\right)}{243}\left(\frac{G_{N} m}{\pi r^{2}}\right)^{4}+O\left(G_{N}^{5}\right) .
\end{aligned}
$$

\section{The metric in the de Donder gauge in six dimensions}

The de Donder gauge condition (5) in $d=5$ reads

$$
8 h_{2}(r)=r \frac{d}{d r}\left(h_{0}(r)+3 h_{1}(r)-h_{2}(r)\right)
$$

supplemented with the asymptotic boundary condition

$$
\lim _{r \rightarrow \infty} f(r)=1 .
$$

This differential equation implies either that $f(r)=C / r$, which does not satisfy the boundary condition (171), or $f(r)$ satisfies the differential equation with $x=G_{N} m /\left(\pi r^{3}\right)$

$$
\begin{aligned}
& x f(x)^{7}\left(6 x-4 f(x)^{3}\right) \frac{d^{2} f(x)}{d x^{2}}+9 f(x)^{6} x^{2}\left(\frac{d f(x)}{d x}\right)^{2}+f(x)^{7}\left(\frac{8}{3} f(x)^{3}-10 x\right) \frac{d f(x)}{d x} \\
& -\frac{5}{3} f(x)^{8}+f(x)^{6}+4 x f(x)^{5}-3 x f(x)^{3}+\frac{9 x^{2}}{4}=0 .
\end{aligned}
$$

We solve the Eq. (172) using a series expansion in $G_{N}$ using (154) and the boundary condition (155). Asking for an expression with only integer powers of $G_{N}$, the result to the order $G_{N}^{7}$ is given by

$$
\begin{aligned}
f(r)= & 1+\frac{G_{N} m}{4 \pi r^{3}}-\frac{5}{8}\left(\frac{G_{N} m}{\pi r^{3}}\right)^{2}+\frac{2}{3}\left(\frac{G_{N} m}{\pi r^{3}}\right)^{3}-\frac{775}{1344}\left(\frac{G_{N} m}{\pi r^{3}}\right)^{4}+\frac{545977}{537600}\left(\frac{G_{N} m}{\pi r^{3}}\right)^{5} \\
& -\frac{15194099}{10483200}\left(\frac{G_{N} m}{\pi r^{3}}\right)^{6}+\frac{4421000509}{1878589440}\left(\frac{G_{N} m}{\pi r^{3}}\right)^{7}+O\left(G_{N}^{8}\right) .
\end{aligned}
$$

The expression is uniquely determined and finite. 


\section{The metric perturbation}

In $d=5$ we derive components of the metric in perturbation by plugging the expression for $f(r)$ in $(173)$ in $(152)$. We obtain for the metric components

$$
\begin{aligned}
& h_{0}^{\mathrm{dD}}(r)=1-\frac{3 G_{N} m}{2 \pi r^{3}}+\frac{9}{8}\left(\frac{G_{N} m}{\pi r^{3}}\right)^{2}-\frac{27}{8}\left(\frac{G_{N} m}{\pi r^{3}}\right)^{3}+\frac{387}{64}\left(\frac{G_{N} m}{\pi r^{3}}\right)^{4}+O\left(G_{N}^{5}\right), \\
& h_{1}^{\mathrm{dD}}(r)=1+\frac{G_{N} m}{2 \pi r^{3}}-\frac{19}{16}\left(\frac{G_{N} m}{\pi r^{3}}\right)^{2}+\frac{49}{48}\left(\frac{G_{N} m}{\pi r^{3}}\right)^{3}-\frac{577}{1344}\left(\frac{G_{N} m}{\pi r^{3}}\right)^{4}+O\left(G_{N}^{5}\right), \\
& h_{2}^{\mathrm{dD}}(r)=\frac{117}{16}\left(\frac{G_{N} m}{\pi r^{3}}\right)^{2}-\frac{45}{16}\left(\frac{G_{N} m}{\pi r^{3}}\right)^{3}+\frac{1599}{112}\left(\frac{G_{N} m}{\pi r^{3}}\right)^{4}+O\left(G_{N}^{5}\right) .
\end{aligned}
$$

\section{RECOVERING THE SCHWARZSCHILD- TANGHERLINI METRIC FROM THE AMPLITUDE COMPUTATIONS}

In this section we show how the amplitude computations match the Schwarzschild-Tangherlini metric in four, five and six dimensions in the de Donder gauge of the previous section.

\section{A. The Schwarzschild metric in four dimensions}

\section{The first post-Minkowskian contribution $\boldsymbol{O}\left(G_{N}\right)$}

Setting $d=3$ in the expressions for the metric perturbation from the tree-level amplitude in (46) matches the de Donder gauge first post-Minkowskian order in four dimension $(d=3)$ in $(160)-(162)$.

\section{The second post-Minkowskian contribution $O\left(G_{N}^{2}\right)$}

At the order $G_{N}^{2}$, setting $d=3$ in the metric perturbation from the one-loop amplitude in (58) matches the metric in the de Donder gauge in four dimensions $(d=3)$ in (160)-(162).

\section{The third post-Minkowskian contributions $O\left(G_{N}^{3}\right)$}

At this order the components of the metric in the de Donder gauge in four dimensions $(d=3)$ from (160)(162) match the metric components from the renormalized two-loop amplitude computation in (120) for the value of the constant of integration

$$
\log C_{3}=\log C_{E}-\frac{7}{2}+6 a^{(1)}(3)
$$

where $C_{E}$ is given in (121).

With this identification we recover the results of [27] for the renormalization of the metric divergences and the coordinate change from the de Donder gauge to the harmonic gauge from the world-line approach.

Substituting this value of $C_{3}$ in the solution (159) completely determines the solution to the de Donder gauge in four dimensions and the coordinate change in (159) to the Schwarzschild metric in (151) in four dimensions.
The parameter $a^{(1)}(3)$ is a free parameter, which corresponds to the running coupling in [27].

\section{The fourth post-Minkowskian contribution $O\left(G_{N}^{4}\right)$}

At the fourth post-Minkowskian order, we get again a diverging metric from the amplitude computation.

This finite component metric in the de Donder gauge in four dimensions $(d=3)$ in (160)-(162) using the value of the constant of integration $C_{3}$ determined in (175) give

$$
\begin{aligned}
& h_{0}^{\mathrm{dD}(4)}=\left(-\frac{32}{3}+8 a^{(1)}(3)+\frac{4}{3} \log \left(\frac{r C_{E}}{G_{N} m}\right)\right)\left(\frac{G_{N} m}{r}\right)^{4}, \\
& h_{1}^{\mathrm{dD}(4)}=\left(10-8 a^{(1)}(3)-\frac{4}{3} \log \left(\frac{r C_{E}}{G_{N} m}\right)\right)\left(\frac{G_{N} m}{r}\right)^{4}, \\
& h_{2}^{\mathrm{dD}(4)}=\left(-\frac{86}{3}+16 a^{(1)}(3)+\frac{8}{3} \log \left(\frac{r C_{E}}{G_{N} m}\right)\right)\left(\frac{G_{N} m}{r}\right)^{4} .
\end{aligned}
$$

This matches exactly the renormalized metric components from the three-loop amplitude computation obtained in (125) with $d=3$.

\section{B. The Schwarzschild-Tangherlini metric in five dimensions}

\section{The first post-Minkowskian contribution $\boldsymbol{O}\left(G_{N}\right)$}

Setting $d=4$ in the expressions for the metric perturbation from the tree-level amplitude in (46) matches the de Donder gauge first post-Minkowskian order in five dimensions $(d=4)$ in (167)-(169).

\section{The second post-Minkowskian contribution $O\left(G_{N}^{2}\right)$}

The renormalized one-loop computation in (130) matches the expression at order $O\left(G_{N}^{2}\right)$ from the de Donder gauge in (167)-(169) for the choice of the constant of integration

$$
\log C_{2}=\frac{11}{15}+2 \log C_{E}+\frac{36 \pi}{5} a^{(1)}(5)
$$


Again there is a free parameter $a^{(1)}(5)$ which can be associated with a running coupling constant.

\section{The third post-Minkowskian contributions $O\left(G_{N}^{3}\right)$}

At the third post-Minkowskian order, we get again a diverging metric from the amplitude computation.

At this order in perturbation, the two-loop amplitude computation had divergences that had to be renormalized to give the in (135). This matches exactly the finite component metric in the de Donder gauge in five dimensions $(d=4)$ in (167)-(169), using the value of the constant of integration $C_{2}$ determined in (177), given by

$$
\begin{aligned}
& h_{0}^{\mathrm{dD}(3)}=\frac{160}{27}\left(\frac{2}{15}+\frac{36 a^{(1)}(5) \pi}{5}+\log \left(\frac{r^{2} C_{E}^{2}}{G_{N} m}\right)\right)\left(\frac{G_{N} m}{\pi r^{2}}\right)^{3}+O(d-4), \\
& h_{1}^{\mathrm{dD}(3)}=-\frac{80}{27}\left(\frac{7}{15}+\frac{36 a^{(1)}(5) \pi}{5}+\log \left(\frac{r^{2} C_{E}^{2}}{G_{N} m}\right)\right)\left(\frac{G_{N} m}{\pi r^{2}}\right)^{3}+O(d-4), \\
& h_{2}^{\mathrm{dD}(3)}=\frac{160}{27}\left(-\frac{1}{15}+\frac{36 a^{(1)}(5) \pi}{5}+\log \left(\frac{r^{2} C_{E}^{2}}{G_{N} m}\right)\right)\left(\frac{G_{N} m}{\pi r^{2}}\right)^{3}+O(d-4) .
\end{aligned}
$$

\section{The fourth post-Minkowskian contribution $O\left(G_{N}^{4}\right)$}

At the fourth post-Minkowskian order, we get again a diverging metric from the amplitude computation.

The three-loop amplitude computation diverges and the finite metric component at the fourth post-Minkowskian order was obtained after normalisation in (142). This matches exactly, the finite component metric in the de Donder gauge in five dimensions $(d=4)$ in (167)-(169), using the value of the constant of integration $C_{2}$ determined in (177), given by

$$
\begin{aligned}
h_{0}^{\mathrm{dD}(4)}= & -\frac{128}{243}\left(23+324 a^{(1)}(5) \pi+45 \log \left(\frac{r^{2} C_{E}^{2}}{G_{N} m}\right)\right)\left(\frac{G_{N} m}{\pi r^{2}}\right)^{4}+O(d-4), \\
h_{1}^{\mathrm{dD}(4)}= & \left(\frac{7085+69552 \pi a^{(1)}(5)+93312\left(\pi a^{(1)}(5)\right)^{2}}{1458}+\frac{10}{243}\left(161+432 \pi a^{(1)}(5)\right) \log \left(\frac{r^{2} C_{E}^{2}}{G_{N} m}\right)\right. \\
& \left.+\frac{100}{81} \log \left(\frac{r^{2} C_{E}^{2}}{G_{N} m}\right)^{2}\right)\left(\frac{G_{N} m}{\pi r^{2}}\right)^{4}+O(d-4), \\
h_{2}^{\mathrm{dD}(4)}= & \left(\frac{-19048-141696 \pi a^{(1)}(5) 373248\left(\pi a^{(1)}(5)\right)^{2}}{729}+\frac{160}{243}\left(-41+216 \pi a^{(1)}(5)\right) \log \left(\frac{r^{2} C_{E}^{2}}{G_{N} m}\right)\right. \\
& \left.+\frac{800}{81} \log \left(\frac{r^{2} C_{E}^{2}}{G_{N} m}\right)^{2}\right)\left(\frac{G_{N} m}{\pi r^{2}}\right)^{4}+O(d-4) .
\end{aligned}
$$

\section{The Schwarzschild-Tangherlini metric in six dimensions}

The metric components in six dimensions $(d=5)$ are finite. They are given up to the order $O\left(G_{N}^{4}\right)$ in (174) and are reproduced by the sum of the contributions of the tree-level amplitude in (46), one-loop amplitude in (58), two-loop amplitude in (76) and three-loop amplitude in (86) and setting $d=5$ in these expressions.

\section{DISCUSSION}

General relativity can be considered in space-times of various dimensions. It is therefore important to validate our current understanding of the connection between scattering amplitudes and classical general relativity in general dimensions [22,23].

We have shown how to reconstruct the classical Schwarzschild-Tangherlini metric from scattering amplitudes in four, five and six dimensions. We have extracted the classical contribution as defined in [12] from the vertex function for the emission of a graviton from a massive scalar field. For such a static metric, the classical contribution is obtained by taking appropriate residues on the time components of the loop momenta. These residues project the quantum scattering amplitude on contribution similar to the quantum tree graphs considered in [7], by cutting the massive propagators. 
The amplitudes develop ultraviolet divergences which are renormalized by introducing higher-derivative nonminimal couplings in (88). The nonminimal coupling removes the ultraviolet divergences in the stress-tensor and the metric components. For the static solution the higher $n \geq 2$ nonminimal coupling only contribute from insertions in tree-level graphs. Interestingly, in six dimensions the metric components are finite but the stresstensor has ultraviolet divergences. These divergences are removed by adding counterterms from nonminimal couplings. These counterterms do not induce any contribution to the metric components. From the presence of ultraviolet poles in the master integrals $J_{(l)}\left(\vec{q}^{2}\right)$ in (32), we conclude that in all dimensions one needs to introduce an infinite set of higher-derivative nonminimal operators for removing the ultraviolet divergences from the scattering amplitude. These counterterms do not affect the space-time geometry because their effect is reabsorbed by the change of coordinate from the de Donder coordinate system to the Schwarzschild-Tangherlini coordinate system.

The scattering amplitude approach presented in this work can be applied to any effective field theory of gravity coupled to matter fields. The amplitudes computations, being performed in general dimensions, lead to results that have an analytic dependence on the spacetime dimensions. As black-hole solutions develop nontrivial properties in general dimensions [25,26], it is interesting to apply the method of this paper to other black-hole metrics. The Kerr-Newman and ReissnerNordström metric in four dimensions have been obtained in [9,41-47] by considering tree-level and one-loop vertex function of the emission of the graviton from a massive particle of spin $s$. The higher order postMinkowskian contributions should be obtained from higher-loop amplitudes in a direct application of the methods used in this work.

\section{ACKNOWLEDGMENTS}

We would like to thank Emil Bjerrum-Bohr, Poul Damgaard, Paolo di Vecchia, Ludovic Planté for discussions and comments. The research of P. Vanhove has received funding from the ANR Grant "Amplitudes" ANR-17- CE31-0001-01, and is partially supported by Laboratory of Mirror Symmetry NRU HSE, RF Government Grant agreement No. 14.641.31.0001. P. V. is grateful to the I.H.E.S. for allowing to use their computer resources.

\section{APPENDIX A: FOURIER TRANSFORMS}

Here we collect the Fourier integrals used to calculate the long range corrections to the energy momentum tensor and the metric.
The Fourier transform form momentum space to direct space

$\mathcal{F}(\alpha, d)=\int_{\mathbb{R}^{d}} \frac{1}{\mid q^{\alpha}} e^{i \vec{q} \cdot \vec{x}} \frac{d^{d} \vec{q}}{(2 \pi)^{d}}=\frac{1}{(4 \pi)^{\frac{d}{2}}} \frac{\Gamma\left(\frac{d-\alpha}{2}\right)}{\Gamma\left(\frac{\alpha}{2}\right)}\left(\frac{2}{|\vec{x}|}\right)^{d-\alpha}$.

Using that

$$
\partial_{x^{i}} \partial_{x^{j}}\left(\vec{x}^{2}\right)^{\alpha}=2 \alpha\left(\vec{x}^{2}\right)^{\alpha-1}\left(\delta_{i j}+2(\alpha-1) \frac{x_{i} x_{j}}{\vec{x}^{2}}\right),
$$

we have that

$$
\begin{aligned}
\mathcal{F}_{i j}(\alpha, d) & :=\int_{\mathbb{R}^{d}} \frac{q_{i} q_{j}}{|\vec{q}|^{\alpha+2}} e^{i \vec{q} \cdot \vec{x}} \frac{d^{d} \vec{q}}{(2 \pi)^{d}} \\
& =\mathcal{F}(\alpha, d)\left(\frac{1}{\alpha} \delta_{i j}+\frac{\alpha-d}{\alpha} \frac{x_{i} x_{j}}{\vec{x}^{2}}\right) .
\end{aligned}
$$

We have in particular that

$$
\mathcal{F}(0, d)=0, \quad \mathcal{F}_{i j}(0, d)=\frac{\Gamma\left(\frac{d}{2}\right)}{2 \pi^{\frac{d}{2}}|\vec{x}|^{d}}\left(\delta_{i j}-d \frac{x_{i} x_{j}}{\vec{x}^{2}}\right) .
$$

\section{APPENDIX B: VERTICES AND PROPAGATORS}

We will here list the Feynman rules which are employed in our calculation. For the derivation of these forms, see [3-5,9,48-50]. Our convention differs from these work by having all incoming momenta. We have stripped off factors of $i \sqrt{8 \pi G_{N}}$ from the vertices and made them explicit in the amplitudes.

(i) The massive scalar propagator is $\frac{i}{q^{2}-m^{2}+i \varepsilon}$.

(ii) The graviton propagator in de Donder gauge can be written in the form $\frac{i \mathcal{P}^{\alpha \beta, \gamma \delta}}{q^{2}+i \varepsilon}$ where $\mathcal{P}^{\alpha \beta, \gamma \delta}$ is defined by

$$
\mathcal{P}^{\mu \nu, \rho \sigma}=\frac{1}{2}\left(\eta^{\mu \rho} \eta^{\nu \sigma}+\eta^{\mu \sigma} \eta^{\nu \rho}-\frac{2}{D-2} \eta^{\mu \nu} \eta^{\rho \sigma}\right)
$$

(i) The 2-scalar-1-graviton vertex $\tau_{1}^{\mu \nu}\left(p_{1}, p_{2}\right)$ is

$$
\tau^{\mu \nu}\left(p_{1}, p_{2}\right)=p_{1}^{\mu} p_{2}^{\nu}+p_{1}^{\nu} p_{2}^{\mu}+\frac{1}{2} \eta^{\mu \nu}\left(p_{1}-p_{2}\right)^{2} .
$$

(ii) The three-graviton vertex has been derived in [49], where $k+q+\pi=0$, 


$$
\begin{aligned}
\tau_{(3)_{\alpha \beta, \gamma \delta}^{\mu \nu}(k, q)=} & -\left(\mathcal{P}_{\alpha \beta \gamma \delta}\left[k^{\mu} k^{\nu}+\pi^{\mu} \pi^{\nu}+q^{\mu} q^{\nu}-\frac{3}{2} \eta^{\mu \nu} q^{2}\right]\right. \\
& +2 q_{\lambda} q_{\sigma}\left[I_{\alpha \beta}{ }^{\sigma \lambda} I_{\gamma \delta}{ }^{\mu \nu}+I_{\gamma \delta}{ }^{\sigma \lambda} I_{\alpha \beta}{ }^{\mu \nu}-I_{\alpha \beta}{ }^{\mu \sigma} I_{\gamma \delta}{ }^{\nu \lambda}-I_{\gamma \delta}{ }^{\mu \sigma} I_{\alpha \beta}{ }^{\nu \lambda}\right] \\
& +\left[q_{\lambda} q^{\mu}\left(\eta_{\alpha \beta} I_{\gamma \delta}{ }^{\nu \lambda}+\eta_{\gamma \delta} I_{\alpha \beta}{ }^{\nu \lambda}\right)+q_{\lambda} q^{\nu}\left(\eta_{\alpha \beta} I_{\gamma \delta}{ }^{\mu \lambda}+\eta_{\gamma \delta} I_{\alpha \beta}{ }^{\mu \lambda}\right)\right. \\
& \left.-q^{2}\left(\eta_{\alpha \beta} I_{\gamma \delta}{ }^{\mu \nu}+\eta_{\gamma \delta} I_{\alpha \beta}{ }^{\mu \nu}\right)-\eta^{\mu \nu} q_{\sigma} q_{\lambda}\left(\eta_{\alpha \beta} I_{\gamma \delta}{ }^{\sigma \lambda}+\eta_{\gamma \delta} I_{\alpha \beta}{ }^{\sigma \lambda}\right)\right] \\
& +\left[2 q_{\lambda}\left(I_{\alpha \beta}{ }^{\lambda \sigma} I_{\gamma \delta \sigma}{ }^{\nu} \pi^{\mu}+I_{\alpha \beta}{ }^{\lambda \sigma} I_{\gamma \delta \sigma}{ }^{\mu} \pi^{\nu}+I_{\gamma \delta}{ }^{\lambda \sigma} I_{\alpha \beta \sigma}{ }^{\nu} k^{\mu}+I_{\gamma \delta}{ }^{\lambda \sigma} I_{\alpha \beta \sigma}{ }^{\mu} k^{\nu}\right)\right. \\
& \left.+q^{2}\left(I_{\alpha \beta \sigma}{ }^{\mu} I_{\gamma \delta}{ }^{\nu \sigma}+I_{\alpha \beta}{ }^{\nu \sigma} I_{\gamma \delta \sigma}{ }^{\mu}\right)+\eta^{\mu \nu} q_{\sigma} q_{\lambda}\left(I_{\alpha \beta}{ }^{\lambda \rho} I_{\gamma \delta \rho}{ }^{\sigma}+I_{\gamma \delta}{ }^{\lambda \rho} I_{\alpha \beta \rho}{ }^{\sigma}\right)\right] \\
& +\left\{\left(k^{2}+\pi^{2}\right)\left[\mathcal{P}_{\alpha \beta}{ }^{\mu \sigma} \mathcal{P}_{\gamma \delta, \sigma}{ }^{\nu}+\mathcal{P}_{\gamma \delta}{ }^{\mu \sigma} \mathcal{P}_{\alpha \beta, \sigma}{ }^{\nu}-\frac{1}{2} \eta^{\mu \nu}\left(\mathcal{P}_{\alpha \beta, \gamma \delta}-\eta_{\alpha \beta} \eta_{\gamma \delta}\right)\right]\right. \\
& \left.\left.+\left(\mathcal{P}_{\gamma \delta}{ }^{\mu \nu} \eta_{\alpha \beta} \pi^{2}+\mathcal{P}_{\alpha \beta}{ }^{\mu \nu} \eta_{\gamma \delta} k^{2}\right)\right\}\right),
\end{aligned}
$$

where $I_{\alpha \beta, \gamma \delta}:=\mathcal{P}_{\alpha \beta, \gamma \delta}+\frac{1}{2} \eta_{\alpha \beta} \eta_{\gamma \delta}$. These vertices are equivalent to the ones computed with the vertices given by De Witt [3-5] and Sannan [50]. We remark that the expression for $\tau_{3}$ is simpler than the three-graviton vertex in these references.

We notice that the three-graviton vertex satisfies the identity

$$
\tau_{(3) \pi \rho, \sigma \tau}^{\mu \nu}(l, q) \mathcal{P}_{\alpha \beta}^{\pi \rho} \mathcal{P}_{\gamma \delta}^{\sigma \tau}=\tau_{(3) \alpha \beta, \gamma \delta}^{\mu \nu}(l, q)
$$

that will be used to simplify the expression of the amplitude.

(iii) The four-graviton vertex with $k_{1}+k_{2}+k_{3}+k_{4}=0$ is given in [31,50]

$$
\begin{aligned}
& \tilde{\tau}_{(4) \mu \nu, \sigma \tau, l \kappa}^{\rho \lambda}\left(k_{1}, k_{2}, k_{3}, k_{4}\right)=-\frac{1}{32}\left(k_{1} \cdot k_{2} \eta^{\mu \nu} \eta^{\sigma \tau} \eta^{\rho \lambda} \eta_{l \kappa}\right)-\frac{1}{16}\left(k_{1}^{\sigma} k_{1}^{\tau} \eta^{\mu \nu} \eta^{\rho \lambda} \eta_{l \kappa}\right) \\
& -\frac{1}{16}\left(k_{1}^{\sigma} k_{2}^{\mu} \eta^{\nu \tau} \eta^{\rho \lambda} \eta_{l \kappa}\right)+\frac{1}{32}\left(k_{1} \cdot k_{2} \eta^{\mu \sigma} \eta^{\nu \tau} \eta^{\rho \lambda} \eta_{l \kappa}\right)+\frac{1}{16}\left(k_{1} \cdot k_{2} \eta^{\mu \nu} \eta^{\sigma \tau} \eta_{l}^{\rho} \eta_{k}^{\lambda}\right) \\
& +\frac{1}{8}\left(k_{1}^{\sigma} k_{1}^{\tau} \eta^{\mu \nu} \eta_{l}^{\rho} \eta_{k}^{\lambda}\right)+\frac{1}{8}\left(k_{1}^{\sigma} k_{2}^{\mu} \eta^{\nu \tau} \eta_{l}^{\rho} \eta_{k}^{\lambda}\right)-\frac{1}{16}\left(k_{1} \cdot k_{2} \eta^{\mu \sigma} \eta^{\nu \tau} \eta_{l}^{\rho} \eta_{k}^{\lambda}\right) \\
& +\frac{1}{4}\left(k_{1} \cdot k_{2} \eta^{\mu \nu} \eta^{\sigma \rho} \eta \tau \lambda \eta_{\iota \kappa}\right)+\frac{1}{4}\left(k_{1}^{\sigma} k_{1}^{\tau} \eta^{\mu \rho} \eta^{\nu \lambda} \eta_{l \kappa}\right)+\frac{1}{8}\left(k_{1}^{\rho} k_{2}^{\lambda} \eta^{\mu \sigma} \eta^{\nu \tau} \eta_{\iota \kappa}\right) \\
& +\frac{1}{2}\left(k_{1}^{\sigma} k_{2}^{\rho} \eta^{\tau \mu} \eta^{\nu \lambda} \eta_{l \kappa}\right)-\frac{1}{4}\left(k_{1} \cdot k_{2} \eta^{\nu \sigma} \eta^{\tau \rho} \eta^{\lambda \mu} \eta_{l \kappa}\right)+\frac{1}{4}\left(k_{1}^{\sigma} k_{2}^{\mu} \eta^{\tau \rho} \eta^{\lambda \nu} \eta_{l \kappa}\right) \\
& +\frac{1}{4}\left(k_{1}^{\sigma} k_{1}^{\rho} \eta^{\tau \lambda} \eta^{\mu \nu} \eta_{l \kappa}\right)-\frac{1}{2}\left(k_{1} \cdot k_{2} \eta^{\mu \nu} \eta^{\tau \rho} \eta_{l}^{\lambda} \eta_{\kappa}^{\sigma}\right)-\frac{1}{2}\left(k_{1}^{\sigma} k_{1}^{\tau} \eta^{\nu \rho} \eta_{l}^{\lambda} \eta_{\kappa}^{\mu}\right) \\
& -\frac{1}{2}\left(k_{1}^{\rho} k_{2}^{\lambda} \eta_{l}^{\nu} \eta_{\kappa}^{\sigma} \eta^{\tau \mu}\right)-\left(k_{1}^{\sigma} k_{2}^{\rho} \eta_{l}^{\tau} \eta_{\kappa}^{\mu} \eta^{\nu \lambda}\right)-\frac{1}{2}\left(k_{1}^{\rho} k_{2 l} \eta^{\lambda \sigma} \eta^{\tau \mu} \eta_{\kappa}^{\nu}\right) \\
& +\frac{1}{4}\left(k_{1} \cdot k_{2} \eta^{\nu \rho} \eta^{\lambda \sigma} \eta_{l}^{\tau} \eta_{\kappa}^{\mu}\right)-\frac{1}{2}\left(k_{1}^{\sigma} k_{1}^{\rho} \eta^{\mu \nu} \eta_{l}^{\tau} \eta_{\kappa}^{\lambda}\right)-\frac{1}{4}\left(k_{1} \cdot k_{2} \eta^{\mu \rho} \eta^{\nu \lambda} \eta_{l}^{\sigma} \eta_{\kappa}^{\tau}\right) \\
& -\frac{1}{2}\left(k_{1}^{\sigma} k_{1}^{\rho} \eta^{\tau \lambda} \eta_{l}^{\mu} \eta_{\kappa}^{\nu}\right)-\frac{1}{4}\left(k_{1}^{\rho} k_{2 l} \eta_{\kappa}^{\lambda} \eta^{\mu \sigma} \eta^{\nu \tau}\right)-\left(k_{1}^{\sigma} k_{2}^{\rho} \eta^{\tau \mu} \eta_{l}^{\nu} \eta_{\kappa}^{\lambda}\right) \\
& -\frac{1}{2}\left(k_{1}^{\sigma} k_{2}^{\mu} \eta^{\tau \rho} \eta_{l}^{\lambda} \eta_{\kappa}^{\nu}\right)+\frac{1}{2}\left(k_{1} \cdot k_{2} \eta^{\nu \sigma} \eta^{\tau \rho} \eta_{l}^{\lambda} \eta_{\kappa}^{\mu}\right)+\operatorname{Sym}\left(k_{1}, k_{2}, k_{3}, k_{4}\right)
\end{aligned}
$$

we introduce the short hand notation

$$
\tau_{(4) \gamma \delta, \sigma \tau, l \theta}^{\mu \nu}\left(k_{1}, k_{2}, k_{3}, k_{4}\right):=\tilde{\tau}_{(4) \alpha \beta, \gamma \delta, \epsilon \eta}^{\mu \nu}\left(k_{1}, k_{2}, k_{3}, k_{4}\right) \mathcal{P}_{\gamma \delta}^{\alpha \beta} \mathcal{P}_{\sigma \tau}^{\gamma \delta} \mathcal{P}_{l \theta}^{\epsilon \eta} .
$$


[1] G. 't Hooft and M. J. G. Veltman, One loop divergencies in the theory of gravitation, Ann. Inst. H. Poincare Phys. Theor. A 20, 69 (1974).

[2] M. J. G. Veltman, Quantum theory of gravitation, Conf. Proc. C 7507281, 265 (1975).

[3] B. S. DeWitt, Quantum theory of gravity. 1. The canonical theory, Phys. Rev. 160, 1113 (1967).

[4] B. S. DeWitt, Quantum theory of gravity. 2. The manifestly covariant theory, Phys. Rev. 162, 1195 (1967).

[5] B. S. DeWitt, Quantum theory of gravity. 3. Applications of the covariant theory, Phys. Rev. 162, 1239 (1967).

[6] J.F. Donoghue, General relativity as an effective field theory: The leading quantum corrections, Phys. Rev. D 50, 3874 (1994).

[7] M. J. Duff, Quantum tree graphs and the Schwarzschild solution, Phys. Rev. D 7, 2317 (1973).

[8] Y. Iwasaki, Quantum theory of gravitation vs classical theory-Fourth-order potential, Prog. Theor. Phys. 46, 1587 (1971).

[9] N. E. J. Bjerrum-Bohr, J. F. Donoghue, and B. R. Holstein, Quantum corrections to the Schwarzschild and Kerr metrics, Phys. Rev. D 68, 084005 (2003).

[10] B. R. Holstein and J. F. Donoghue, Classical Physics and Quantum Loops, Phys. Rev. Lett. 93, 201602 (2004).

[11] J. F. Donoghue and T. Torma, On the power counting of loop diagrams in general relativity, Phys. Rev. D 54, 4963 (1996).

[12] N. E. J. Bjerrum-Bohr, P. H. Damgaard, G. Festuccia, L. Planté, and P. Vanhove, General Relativity from Scattering Amplitudes, Phys. Rev. Lett. 121, 171601 (2018).

[13] D. A. Kosower, B. Maybee, and D. O'Connell, Amplitudes, observables, and classical scattering, J. High Energy Phys. 02 (2019) 137.

[14] C. Cheung, I. Z. Rothstein, and M. P. Solon, From Scattering Amplitudes to Classical Potentials in the Post-Minkowskian Expansion, Phys. Rev. Lett. 121, 251101 (2018).

[15] Z. Bern, C. Cheung, R. Roiban, C. H. Shen, M. P. Solon, and M. Zeng, Scattering Amplitudes and the Conservative Hamiltonian for Binary Systems at Third Post-Minkowskian Order, Phys. Rev. Lett. 122, 201603 (2019).

[16] Z. Bern, C. Cheung, R. Roiban, C. H. Shen, M. P. Solon, and M. Zeng, Black hole binary dynamics from the double copy and effective theory, J. High Energy Phys. 10 (2019) 206.

[17] M. Z. Chung, Y. T. Huang, and J. W. Kim, Classical potential for general spinning bodies, J. High Energy Phys. 09 (2020) 074.

[18] G. Kälin and R. A. Porto, From boundary data to bound states, J. High Energy Phys. 01 (2020) 072.

[19] C. Cheung and M. P. Solon, Classical gravitational scattering at $\mathcal{O}\left(G^{3}\right)$ from Feynman diagrams, J. High Energy Phys. 06 (2020) 144.

[20] G. Mogull, J. Plefka, and J. Steinhoff, Classical black hole scattering from a worldline quantum field theory, arXiv: 2010.02865 .

[21] A. K. Collado, P. Di Vecchia, R. Russo, and S. Thomas, The subleading eikonal in supergravity theories, J. High Energy Phys. 10 (2018) 038.

[22] A. Koemans Collado, P. Di Vecchia, and R. Russo, Revisiting the second post-Minkowskian Eikonal and the dynamics of binary black holes, Phys. Rev. D 100, 066028 (2019).

[23] A. Cristofoli, P. H. Damgaard, P. Di Vecchia, and C. Heissenberg, Second-order post-Minkowskian scattering in arbitrary dimensions, J. High Energy Phys. 07 (2020) 122.

[24] G. U. Jakobsen, Schwarzschild-Tangherlini metric from scattering amplitudes, J. High Energy Phys. 02 (2019) 137.

[25] R. Emparan and H. S. Reall, Black holes in higher dimensions, Living Rev. Relativity 11, 6 (2008).

[26] R. Emparan, R. Suzuki, and K. Tanabe, The large D limit of general relativity, J. High Energy Phys. 06 (2013) 009.

[27] W. D. Goldberger and I. Z. Rothstein, An effective field theory of gravity for extended objects, Phys. Rev. D 73, 104029 (2006).

[28] N. E. J. Bjerrum-Bohr, J. F. Donoghue, and B. R. Holstein, On the parameterization dependence of the energy momentum tensor and the metric, Phys. Rev. D 75, 108502 (2007).

[29] P. Fromholz, E. Poisson, and C. M. Will, The Schwarzschild metric: It's the coordinatesstupid!, Am. J. Phys. 82, 295 (2014).

[30] Citation for the 2020 Nobel prize award to Roger Penrose, https://www.nobelprize.org/prizes/physics/2020/ press-release/.

[31] L. Planté, Some aspects on effective field theory of gravity, Ph.D. thesis, Université Paris-Saclay, 2016.

[32] J. Galusha, Investigations into the classical limit of quantum field theory, Master thesis Niels Bohr International Academy, 2018.

[33] M. Levy and J. Sucher, Eikonal approximation in quantum field theory, Phys. Rev. 186, 1656 (1969).

[34] R. N. Lee, Presenting litered: A tool for the loop integrals reduction, arXiv:1212.2685.

[35] R. N. Lee, LiteRed 1.4: A powerful tool for reduction of multiloop integrals, J. Phys. Conf. Ser. 523, 012059 (2014).

[36] P. Vanhove, The physics and the mixed hodge structure of Feynman integrals, Proc. Symp. Pure Math. 88, 161 (2014).

[37] A. Cristofoli, N. E. J. Bjerrum-Bohr, P. H. Damgaard, and P. Vanhove, Post-Minkowskian Hamiltonians in general relativity, Phys. Rev. D 100, 084040 (2019).

[38] J. C. Lagarias, Euler's constant: Euler's work and modern developments, Bull. Am. Math. Soc. (N.S.) 50, 527 (2013).

[39] D. Prinz, Gravity-Matter Feynman rules for any valence, arXiv:2004.09543.

[40] F. R. Tangherlini, Schwarzschild field in $N$ dimensions and the dimensionality of space problem, Nuovo Cimento 27, 636 (1963).

[41] J. F. Donoghue, B. R. Holstein, B. Garbrecht, and T. Konstandin, Quantum corrections to the ReissnerNordstrom and Kerr-Newman metrics, Phys. Lett. B 529, 132 (2002); Erratum, Phys. Lett. B 612, 311 (2005).

[42] A. Guevara, A. Ochirov, and J. Vines, Scattering of spinning black holes from exponentiated soft factors, J. High Energy Phys. 09 (2019) 056.

[43] M. Z. Chung, Y. T. Huang, J. W. Kim, and S. Lee, The simplest massive s-matrix: From minimal coupling to black holes, J. High Energy Phys. 04 (2019) 156. 
[44] N. Moynihan, Kerr-Newman from minimal coupling, J. High Energy Phys. 01 (2020) 014.

[45] M. Z. Chung, Y. T. Huang, and J. W. Kim, Kerr-Newman stress-tensor from minimal coupling to all orders in spin, arXiv:1911.12775.

[46] A. Guevara, A. Ochirov, and J. Vines, Black-hole scattering with general spin directions from minimal-coupling amplitudes, Phys. Rev. D 100, 104024 (2019).

[47] A. Cristofoli, Gravitational shock waves and scattering amplitudes, J. High Energy Phys. 11 (2020) 160.
[48] N. E. J. Bjerrum-Bohr, J. F. Donoghue, and P. Vanhove, On-shell techniques and universal results in quantum gravity, J. High Energy Phys. 02 (2014) 111.

[49] J. F. Donoghue, Introduction to the effective field theory description of gravity, in Advanced School on Effective Theories Almunecar, Spain, 1995 (World Scientific Publishing Co, 1995).

[50] S. Sannan, Gravity as the limit of the type II superstring theory, Phys. Rev. D 34, 1749 (1986). 